\title{
TANGENT SCROLLS IN PRIME FANO THREEFOLDS
}

\author{
Atanas Iliev and Carmen Schuhmann
}

\begin{abstract}
In this paper we prove that any smooth prime Fano threefold, different from the Muka1-Umemura threefold $X_{22}^{\prime}$, contains a 1-dimensional family of intersecting lines. Combined with a result in $[\mathrm{Sch}]$ this implies that any morphism from a smooth Fano threefold of index 2 to a smooth Fano threefold of index 1 must be constant, which gives an answer in dimension 3 to a question stated by Peternell.
\end{abstract}

\section{\$1. Introduction}

1.1. A smooth projective variety $X$ is called a Fano variety if the anticanonical bundle $-K_{X}$ is ample. Then the index of $X$ is the largest positive integer $r=r(X)$ such that $-K_{X}=r H$ for some line bundle $H$ on $X$.

The smooth Fano threefold $X=X_{d} \subset \boldsymbol{P}^{g+1}(d=\operatorname{deg} X)$ is called prime if $\rho(X)=\operatorname{rank} \operatorname{Pic}(X)=1, r(X)=1$, and $-K_{X}$ is the hyperplane bundle on $X$. By the classification of Fano threefolds smooth prime Fano threefolds exist iff $3 \leq$ $g \leq 12(g \neq 11)$, and then $d=2 g-2$ (see [I1]).

1.2. (see $\S 4.2, \S 4.4$ in [IP], or $\S 1$ in [I2]). Let $l$ be a line on the smooth prime Fano threefold $X$, and let $N_{l / X}$ be the normal bundle of $l \subset X$. Then

(1). either (a). $\quad N_{l / X}=\mathcal{O} \oplus \mathcal{O}(-1)$; or (b). $\quad N_{l / X}=\mathcal{O}(1) \oplus \mathcal{O}(-2)$.

(2). The Hilbert scheme $\mathscr{H}_{X}$ of lines on $X$ is non-empty, any irreducible component $\mathscr{H}_{o}$ of $\mathscr{H}_{X}$ is one-dimensional, and either $\mathscr{H}_{o}$ is non-exotic, i.e. $N_{l / X}$ is of type (1)(a) for the general $l \in \mathscr{H}_{o}$; or $\mathscr{H}_{o}$ is exotic, i.e. $N_{l / X}$ is of type (1)(b) for any $l \in \mathscr{H}_{0}$.

(3). The component $\mathscr{H}_{0}$ is exotic if either the elements $l \in \mathscr{H}_{0}$ sweep out the tangent scroll $R_{o} \subset X$ to an irreducible curve $C \subset X$; or $g=3$ (i.e. $X=X_{4}$ is a quartic threefold), and then the lines $l \in \mathscr{H}_{o}$ sweep out a hyperplane section $R_{o} \subset$ $X_{4}$ which is a cone over a plane quartic curve, centered at some point $x \in X_{4}$.

1.3. For example, the scheme $\mathscr{H}_{X}$ of the Fermat quartic $X=X_{4}=$ $\left(x_{0}^{4}+\cdots+x_{4}^{4}=0\right)$, which is a prime Fano threefold of $g=3$, is a union of 40 double components each of which is of type (1.2)(1)(b) (see Remark 3.5(ii) in [I1]).

Received September 7, 1999; revised March 21, 2000. 
The only known example of a prime Fano threefold $X$ of $g \geq 4$ such that $\mathscr{H}_{X}$ has an exotic component $\mathscr{H}_{o}$, is the Mukai-Umemura threefold $X_{22}^{\prime}$. The scheme $\mathscr{H}_{X_{22}^{\prime}}=2 \mathscr{H}_{o}$ and the surface $R_{o}$ is the hyperplane section of $X_{22}^{\prime}$ swept out by the tangent lines to a rational normal curve $C_{12} \subset X_{22}^{\prime}$ of degree 12 (see [MU]).

1.4. By a theorem of Kobayashi and Ochiai the index $r=r(Y)$ of a smooth Fano $n$-fold $Y$ can't be greater than $n+1$; and the only smooth Fano $n$-folds of $r \geq n$ are $\boldsymbol{P}^{n}$ for which $r=n+1$ and the $n$-dimensional quadric $Q_{2}^{n}$ for which $r=$ $n$ (see e.g. $[\mathrm{Pe}]$, p. 106). In particular, except $\boldsymbol{P}^{3}$ and $Q_{2}^{3}$, any smooth Fano 3fold must have index $r \leq 2$.

It is shown by Remmert and Van de Ven (for $n=2$ ) and later by Lazarsfeld (for any $n$ ) that the projective space $\boldsymbol{P}^{n}$ does not admit surjective morphisms to a smooth projective $n$-fold $X \neq \boldsymbol{P}^{n}$ (see [RV], [L]). The same is true for morphisms $f: Q_{2}^{n} \rightarrow X \neq \boldsymbol{P}^{n}, Q_{2}^{n}$ (see [PS]). In particular, $\boldsymbol{P}^{3}$ and $Q_{2}^{3}$ do not admit surjective moprphisms to smooth Fano threefolds $X$ of smaller index $r(X)$.

Let $f: Y \rightarrow X$ be a non-constant morphism between smooth Fano 3-folds of $\rho=1$. By Kor. 1.5 in [RV], $\rho(Y)=1$ implies that $f$ must be surjective, and by the preceding $r(Y)$ can't be $\geq 3$. Therefore $r(Y)=2, r(X)=1$. This gives rise to the following question stated originally by Peternell (see $(2.12)(2)$ in $[\mathrm{Pe}])$.

QUESTION (Pe). Are there non-constant (hence surjective) morphisms $f$ : $Y \rightarrow X$ from a smooth Fano 3-fold $Y$ of $\rho(Y)=1$ and $r(Y)=2$ to a smooth Fano 3-fold $X$ of $\rho(X)=1$ and $r(X)=1$ ?

In this paper we give the expected negative answer to $(\mathrm{Pe})$.

Let $f: Y \rightarrow X$ be as above, and assume that $f$ is non-constant. Then $f$ must be surjective and finite since $\rho(Y)=1$ (see Kor. 1.5 in [RV]). Therefore $f^{*}: H^{3}(X, C) \rightarrow H^{3}(Y, C)$ will be an embedding, in particular $h^{3}(X) \leq h^{3}(Y)$ (see also [Sch]). For any Fano threefold $h^{3,0}=0$ and $h^{3}=2 h^{2,1}$ since the anticanonical class is ample. Therefore $h^{2,1}(X) \leq h^{2,1}(Y)$. Since $h^{2,1}(Y) \leq 21$ for any Fano 3-fold $Y$ of $r=2$ (see [I1]), then the existence of a non-constant morphism $f: X \rightarrow Y$ as in $(\mathrm{Pe})$ implies that $h^{2,1}(X) \leq 21$. This gives a negative answer to $(\mathrm{Pe})$ whenever $h^{2,1}(X)>21$.

The only smooth non-prime Fano threefolds of $\rho=1$ and $r=1$ are the sextic double solid $X_{2}^{\prime}$ and the double quadric $X_{4}^{\prime}$ for which the answer to $(\mathrm{Pe})$ is negative since $h^{2,1}\left(X_{2}^{\prime}\right)=52>21$ and $h^{2,1}\left(X_{4}^{\prime}\right)=30>21$. By the same argument the answer to (Pe) is negative also for the quartic threefold $X_{4}$ since $h^{2,1}\left(X_{4}\right)=30>21$. Any other smooth prime Fano threefold $X=X_{2 g-2} \subset \boldsymbol{P}^{g+1}$, $4 \leq g \leq 12, g \neq 11$ has $h^{2,1}(X) \leq 20$ (see [I1]).

In $[\mathrm{Sch}]$ is given a negative answer to $(\mathrm{Pe})$ provided $X$ contains a conic of rank 2 (a pair of intersecting lines). The only known Fano threefold $X$ of $\rho(X)=1$ and $r(X)=1$ without intersecting lines is the Mukai-Umemura threefold $X=X_{22}^{\prime}$, and a negative answer to $(\mathrm{Pe})$ in case $X=X_{22}^{\prime}$ is given by $\mathrm{E}$. Amerik (see $[\mathrm{Sch}])$. 
Therefore, in order to give a negative answer to $(\mathrm{Pe})$, it is enough to prove the following

Proposition (B). Any smooth prime Fano threefold $X_{2 g-2} \subset \boldsymbol{P}^{g+1}, 4 \leq$ $g \leq 12, g \neq 11$, different from the Mukai-Umemura threefold $X_{22}^{\prime}$, contains a 1dimensional family of conics of rank 2 .

In Section 2 we prove Proposition (B) for $4 \leq g \leq 9$ on the base of the following technical

Lemma (A). A smooth prime Fano threefold $X=X_{2 g-2} \subset \boldsymbol{P}^{g+1}, 3 \leq g \leq 9$ can't contain the tangent scroll $S_{2 g-2}$ to a rational normal curve $C_{g}$ of degree $g$.

By a result of $\mathrm{Yu}$. Prokhorov, the only smooth prime $X=X_{2 g-2}, g=10,12$ such that the scheme of lines $\mathscr{H}_{X}$ has an exotic component is the MukaiUmemura threefold $X_{22}^{\prime}$ (see [Pr]). This implies Proposition (B) for $g=10,12$. Indeed, let $X=X_{2 g-2}$ be a smooth prime Fano threefold such that the scheme of lines $\mathscr{H}_{X}$ on $X$ has a non-exotic component $\mathscr{H}_{o}$. Then, by Lemma 3.7 in [I1], the general element of $\mathscr{H}_{o}$ will represent a line $l \subset X$ which intersects at least one other line on $X$.

This completes the proof of Proposition (B), which yields a negative answer to $(\mathrm{Pe})$.

In Section 3 we prove Lemma (A) for any particular value of $g, 3 \leq g \leq 9$.

For the prime Fano threefolds $X_{2 g-2} \subset \boldsymbol{P}^{g+1}(g=3,4,5,6,8)$ we prove Lemma (A) by using the Mukai's representation (see (3.1)) of the smooth $X_{2 g-2}$, $3 \leq g \leq 10$ as a complete intersection in a homogeneous or almost-homogeneous variety $\Sigma(g)$. More concretely we see that if the threefold $X \subset \Sigma(g)(g=3,4$, $5,6,8)$ is a complete intersection in $\Sigma(g)$ of the same type as the smooth prime $X_{2 g-2}$, and if $X$ contains the tangent scroll $S_{2 g-2}$ to the rational normal curve $C_{g}$ of degree $g$, then $X$ must be singular-see (3.4), (3.5), (3.6), (3.13), (3.15), (3.19).

For $g=7$ we use the properties of the projection from a special line $l \subset$ $X_{2 g-2}, g \geq 7$ to reduce the proof of Lemma (A) for $g=7$ to the already proved Lemma (A) for $g=5$ - see (3.20)-(3.24). To prove Lemma (A) in case $g=9$ we can use the same approach as for $g=7$. But a more elegant proof, based on the description of the double projection from a line, had been suggested by the Referee-see (3.25).

\section{§2. Lemma (A) $\Rightarrow$ Proposition (B) for $4 \leq g \leq 9$.}

2.0. Assume that the smooth prime Fano threefold $X=X_{2 g-2}(4 \leq g \leq 9)$ does not contain a 1-dimensional family of conics of rank 2 .

LEMMA 2.1. Under the assumption (2.0):

(i). The Hilbert scheme $\mathscr{H}_{X}$ of lines on $X$ has a unique irreducible component $\mathscr{H}_{o}$; 
(ii). $\mathscr{H}_{o}=\mathscr{H}_{X}$ is exotic, and the lines $l \in \mathscr{H}_{o}$ sweep out a tangent scroll $R_{o} \in$ $\left|\mathcal{O}_{X}(d)\right|, d \geq 2$.

Proof of Lemma 2.1.

(i). Let $\mathscr{H}_{o}$ and $\mathscr{H}_{\infty}$ be two different irreducible components of $\mathscr{H}_{X}$, and let $R_{o}$ and $R_{\infty}$ be the surfaces swept out by the lines $l \in \mathscr{H}_{o}$ and $l \in \mathscr{H}_{\infty}$. Since $\operatorname{Pic}(X)=Z$. $H$, where $H$ is the hyperplane section, any effective divisor on $X$ must be ample. In particular the general line $l \in \mathscr{H}_{0}$ intersects the surface $R_{\infty} \subset X$. If moreover $l \subset R_{\infty}$ for the general $l \in \mathscr{H}_{o}$, then $R_{o}=R_{\infty}$ and this surface contains two 1-dimensional families of lines. Therefore $R_{o}=R_{\infty}$ is a quadric surface on $X$, which contradicts $\operatorname{Pic}(X)=Z$. $H$. Therefore the general $l \in \mathscr{H}_{o}$ intersects $R_{\infty}$ and does not lie in $R_{\infty}$; and since $R_{\infty}$ is swept out by lines then there exists a line $l^{\prime} \subset R_{\infty}$ which intersects $l$. Since $l \in \mathscr{H}_{0}$ is general this produces a 1-dimensional family of intersecting lines $l+l^{\prime}(=$ conics of rank 2 on $X$ )-contradiction.

(ii). If $\mathscr{H}_{X}=\mathscr{H}_{o}$ is non-exotic then, by Lemma 3.7 of [I1], the general element of $\mathscr{H}_{0}$ will represent a line $l \subset X$ which will intersect at least one other line $m \subset X$, i.e. $X$ will contain a 1-dimensional family of intersecting lines (= conics of rank 2). Therefore $\mathscr{H}_{0}$ is exotic.

Since $\mathscr{H}_{o}$ is exotic and $g \geq 4$ then $R_{o}$ is the tangent scroll to a curve $C \subset X$ (see (1.2)(3)), and since $\operatorname{Pic}(X)=Z . H$ then $R_{o} \in\left|\mathcal{O}_{X}(d)\right|$ for some ineteger $d \geq 1$. If $R_{o}$ is a hyperplane section of $X$ (i.e. $d=1$ ) then, by Lemma 6 in [Pr], $C=C_{g}$ must be a rational normal curve of degree $g$. However the last is impossible since then Lemma (A) will imply that $X$ is singular. Therefore $d \geq 2$. q.e.d.

We shall show that nevertheless $X$ contains a 1-dimensional family of conics $l+m$ of rank 2 where $l, m \in \mathscr{H}_{o}$.

Remark. Let $C_{s}$ be the (possibly empty) set of singular points of $C$. For any $x \in C-C_{s}$ denote by $l_{x}$ the tangent line to $C$ at $x$. For a point $x=x(0) \in$ $C_{s}$ define a tangent line to $C$ at $x$ to be any $\operatorname{limit}_{\lim _{x(t) \rightarrow x(0)}} l_{x(t)}$ of tangent lines $l_{x(t)}$ to points $x(t) \in C-C_{s}$ (see Chapter $2 \S 4$ in $[\mathrm{GH}]$ ). Clearly, $C$ can have only a finite number of tangent lines to $x(0) \in C_{s}$ (see also Chapter $2 \S 1.5$ in [Sh]).

2.2. By the initial assumption (2.0), $X$ does not contain a 1-dimensional family of conics of rank 2. In particular, $X$ does not contain a 1-dimensional family of pairs of intersecting tangent lines to $C$.

Since the surface $R_{o} \in\left|\mathcal{O}_{X}(d)\right|, d \geq 2$, and $\operatorname{Span} X=\boldsymbol{P}^{g+1}$ then $\operatorname{Span} R_{o}=$ $\boldsymbol{P}^{g+1}, g \geq 4$ (see also Lemma 6 in $[\operatorname{Pr}]$ ). Since $R_{o}$ is swept out by the tangent lines to $C$ then $\operatorname{Span} C=\operatorname{Span} R_{o}=\boldsymbol{P}^{g+1}$. In particular $C$ does not lie on a plane. Since $R_{o}$ is the tangent scroll to the non-plane curve $C$ then $S_{C}$ is singular along the curve $C$.

Let $L \neq C$ be (if exists) an irreducible curve on $R_{o}$ such that $R_{o}$ is singular along $L$. If $L$ is not a tangent line to $C$, then the general point of $L$ will be an intersection point of two or more tangent lines to $C$ (see $\S 4$ in [P2]). The last is 
impossible since, by assumption, on $X$ can lie at most a finite number of pairs of intersecting lines.

Therefore any irreducible curve $L \neq C$ such that $L \subset \operatorname{Sing} R_{o}$ must be a tangent line to $C$. In addition, the tangent scroll $R_{o}$ to $C$ still can be singular along a tangent line $L$ to $C$-for example if $L$ is a common tangent line to two or more branches of $C$ at $x$, or if $C$ has a branch with a cusp at $x$, or if $x \in$ $C-C_{s}$ but $x$ is an inflexion point of $C$ and then $R_{o}$ has a cusp along $l_{x}$, etc. (see $\S 2, \S 4$ in $[\mathrm{P} 2])$.

Let $\Delta \subset R_{o}$ be the union of all the irreducible curves $L$ on $R_{o}$ such that $L \neq C$ and $R_{o}$ is singular along $L$. By the above argument, either $\Delta=\emptyset$ or $\Delta$ is a union of a finite number of tangent lines to $C$.

Let $v: R_{n} \rightarrow R_{o}$ be the normalization of $R_{o}$. Fix a desingularization $\tau$ : $\tilde{R}_{n} \rightarrow R_{n}$, and let $\sigma=\tau \circ v: \tilde{R}_{n} \rightarrow R_{o}$. Let $E_{1}, \ldots, E_{k}(k \geq 0)$ be all the irreducible contractable curves on $\tilde{R}_{n}$, i.e. all the irreducible curves $E_{l} \subset \tilde{R}_{n}$ such that $\sigma\left(E_{l}\right) \in R_{o}$ is a point.

Denote by $\sim$ the linear equivalence of divisors on the smooth surface $\tilde{R}_{n}$, and let $E$ be a divisor on $\tilde{R}_{n}$. Call $E$ a zero divisor on $\tilde{R}_{n}$ if $E \sim 0$; call the nonzero divisor $E$ contractable if $E \sim a_{1} E_{1}+\cdots+a_{k} E_{k}$ for some $a_{1}, \ldots, a_{k} \in Z$. Let $C^{\prime} \subset \tilde{R}_{n}$ be the proper $\sigma$-preimage of $C$ on $\tilde{R}_{n}$. Since $R_{o}$ is the tangent scroll to the irreducible curve $C$ then the curve $C^{\prime}$ is irreducible and $\left.\sigma\right|_{C^{\prime}}: C^{\prime} \rightarrow C$ is an isomorphism over a dense open subset of $C$ (see also Lemma 2.3 below).

Let $C_{1}^{\prime}, \ldots, C_{r}^{\prime}$ be all the irreducible curves on $\tilde{R}_{n}$ such that $\sigma\left(C_{i}^{\prime}\right)$ is an irreducible component of $\Delta$. Therefore

$$
K_{\tilde{R}_{n}} \sim \sigma^{*} K_{R_{o}}-m C^{\prime}-\Sigma_{l=1, \ldots, r} p_{i} C_{i}^{\prime}+E
$$

for some positive integers $m, p_{1}, \ldots, p_{r}$, and a contractable (or zero) divisor $E$ on $\tilde{R}_{n}$.

Lemma 2.3. Let $X$ fulfills (2.0). Then the tangent scroll $R_{o} \subset X$ to $C$ has a cusp of type $v^{2}=u^{3}+\cdots$ along $C$, at a neighbourhood of the general point $x \in C \subset R_{o}$; in particular $m=$ mult $_{C} R_{o}=2$ (see also $\S 5$ in [P2] and $\S 4$ in [P1]).

\section{Proof of Lemma 2.3.}

(1). We shall see first that $R_{o}$ is irreducible at any neighbourhood of the general point $x \in C$, i.e. $R_{o}$ has one local branch at $x$.

Assume the contrary, and let $x \in C$ be general. Let $U \subset X$ be a complexanalytical neighbourhood of $x$ such that $R_{U}=R_{o} \cap U$ is reducible. Since $R_{o}$ is swept out by the tangent lines to $C$, the last imlies that for the general point $y \in C_{U}=C \cap U$ (hence for the general $y \in C$ ) there exists (possibly non-unique) $z \in C, z \neq y$ such that $y$ lies on a tangent line to $C$ at $z$. Since the set $C_{s}=$ $\left\{x_{1}, \ldots, x_{s}\right\}$ of singular points of $C$ is finite (or empty), and any such $x_{l}$ has at most a finite number of tangent lines, then the general $y \in C$ doesn't lie on a tangent line to $z \in C_{s}$. Therefore the general $y \in C$ lies on the tangent line $l_{z}$ to $C$ at some (possibly non-unique) $z \in C-C_{s}$.

If moreover $l_{y} \neq l_{z}$ (where $l_{y}$ is the tangent line to $C$ at $y$ ) then all such $l_{y}+l_{z}$ 
will produce a 1-dimensional family of conics of rank 2 on $X$, which contradicts the initial assumption (2.0) about $X$.

If $l_{y}=l_{z}$ then this will imply that the tangent line $l_{y}$ to $C$ at the general $y \in C$ is tangent to $C$ at two or more points. But then the projection $\bar{C}$ of $C \subset \boldsymbol{P}^{g+1}$ from the general subspace $\boldsymbol{P}^{g-2} \subset \boldsymbol{P}^{g+1}=\operatorname{Span} X$ will be a plane curve with a 1dimensional family of lines tangent to $C$ at two or more points, which is impossible.

(2). It rests to see that the unique local branch of $R_{o}$ at the general $x \in C$ has a cusp of type $v^{2}=u^{3}+\cdots$ along $C$ at a neighbourhood of $x$. above).

Since $R_{o} \in\left|\mathcal{O}_{X}(d)\right|$ and $d \geq 2$, then $\operatorname{Span} C=\operatorname{Span} R_{o}=\boldsymbol{P}^{g+1}, g \geq 4$ (see

Let $x$ be a general point of $C$. In order to prove that the tangent scroll $R_{o} \subset X$ to $C$ has a cusp of type $v^{2}=u^{3}+\cdots$ at a neighbourhood of $x$ it is enough to see that the projection of $R_{o}$ from a general $\boldsymbol{P}^{g-3} \subset \boldsymbol{P}^{g+1}$ has a cusp at $x$. This reduces the check to the case when $R_{o}$ is the tangent scroll to a curve $C \subset \boldsymbol{P}^{3}$.

Since $x$ is a general point of $C \subset \boldsymbol{P}^{3}$ then, after a possible linear change of coordinates in $\boldsymbol{P}^{3}$, the curve $C$ has (at $\left.x=(1: 0: 0: 0)\right)$ a local parameterization, or a normal form (see $\S 2$ in [P2], or Chapter $2 \S 4$ in $[\mathrm{GH}]$ ):

$$
C_{U}:\left(x_{o}(z): \cdots: x_{n}(z)\right)=\left(1: z+o\left(z^{2}\right): z^{2}+o\left(z^{3}\right): z^{3}+o\left(z^{4}\right)\right), \quad|z|<1,
$$

where $o\left(z^{k}\right)=\Sigma_{j \geq k} a_{j} z^{j}$. Since the coefficient at $z^{k}$ in $x_{k}(z)=z^{k}+o\left(z^{k+1}\right)$ is $1 \neq 0 \quad(k=2,3)$ then, after a possible linear change of $\left(x_{1}, x_{2}, x_{3}\right)$, the local parameterization of $C$ at $x=(1: 0: 0: 0)$ can be written as

$$
\begin{aligned}
C_{U}: & \left(x_{o}(z): x_{1}(z): x_{2}(z): x_{3}(z)\right) \\
& =\left(1: z+o\left(z^{4}\right): z^{2}+o\left(z^{4}\right): z^{3}+o\left(z^{4}\right)\right), \quad|z|<1,
\end{aligned}
$$

i.e. $C_{U}$ approximates, upto $o\left(z^{4}\right)$, the twisted cubic $C_{3}=\left\{\left(1: z: z^{2}: z^{3}\right)\right\}$. Therefore, at a neighbourhood of $x=(1: 0: 0: 0)$, the unique local branch (see (1)) of the tangent scroll $R_{o}$ to $C$ is parameterized by

$$
\begin{aligned}
R_{U}:( & \left.x_{o}(z, t): x_{1}(z, t): x_{2}(z, t): x_{3}(z, t)\right) \\
= & \left(1: z+t+o\left(z^{4}\right)+o\left(z^{3}\right) t: z^{2}+2 z t+o\left(z^{4}\right)\right. \\
& \left.+o\left(z^{3}\right) t: z^{3}+3 z^{2} t+o\left(z^{4}\right)+o\left(z^{3}\right) t\right) .
\end{aligned}
$$

In affine coordinates $\left(x_{1}, x_{2}, x_{3}\right)$ the tangent line to $C$ at $x=(0,0,0)$ is spanned by the vector $n_{x}=(1,0,0)$, and the normal space $C_{o}^{2} \subset C^{3}\left(x_{1}, x_{2}, x_{3}\right)$ to $n_{x}$ at $x$ is defined by $x_{1}=0$. In order to prove that $R_{U}$ has a cusp along $C$ at a neighbourhood of $x$ we shall see that the curve $D_{U}=R_{U} \cap\left(x_{1}=0\right) \subset C_{o}^{2}$ has a cusp at $x$.

On $D_{U}=R_{U} \cap\left(x_{1}=0\right)$, one has: $0=x_{1}=z+t+o\left(z^{4}\right)+o\left(z^{3}\right) t$, i.e. $t=$ $-z+o\left(z^{4}\right)$. Let $u=-x_{2}, v=-x_{3} / 2$. Therefore, on $D_{U} \subset C_{o}^{2}$ 


$$
\begin{gathered}
u=-\left(z^{2}+2 z t+o\left(z^{4}\right)+o\left(z^{3}\right) t\right)=z^{2}+o\left(z^{4}\right) \\
v=-1 / 2\left(z^{3}+3 z^{2} t+o\left(z^{4}\right)+o\left(z^{3}\right) t\right)=z^{3}+o\left(z^{4}\right),
\end{gathered}
$$

i.e. $u^{2}=z^{4}+o\left(z^{6}\right), u v=z^{5}+o\left(z^{6}\right), v^{2}=z^{6}+o\left(z^{7}\right), u^{3}=z^{6}+o\left(z^{8}\right), u^{2} v=z^{7}+$ $o\left(z^{8}\right), \ldots$

Let $\left.C\right|_{U}=\left(f_{U}(u, v)=0\right)$ be the local equation of $D_{U} \subset C_{o}^{2}(u, v)$ at $x=$ $(0,0)$. Therefore, upto a constant non-zero factor, $f_{U}(u, v)=v^{2}-u^{3}+c_{2,1} u^{2} v+$ $c_{1,2} u v^{2}+\cdots$, i.e. $\left.C\right|_{U}$ has a double cusp-singularity of type $v^{2}=u^{3}+\cdots$ at $x=$ $(0,0)$ (see $\S 5$ in $[\mathrm{P} 1], \S 4$ in [P2], Chapter 5 Examples 3.9.5, 3.9.1 and Chapter 1 Exercise 5.14 in $[\mathrm{H}])$.

Therefore $R=R_{o}$ has a pinch of type $v^{2}=u^{3}+\cdots$ along $C$ at a neighbourhood of the general point $x \in C$, which proves Lemma 2.3.

2.4. By the definition of $C_{1}^{\prime}, \ldots, C_{r}^{\prime}$ any irreducible component of $\Delta$ can be represented (possibly non-uniquely) as the image $\sigma\left(C_{i}^{\prime}\right)$ of some $C_{i}^{\prime}, i=1, \ldots, r$. Since $\left.\sigma\right|_{C^{\prime}}: C^{\prime} \rightarrow C$ is an isomorphism over an open dense subset of $C$ then the general point $x \in C$ has a unique $\sigma$-preimage $x^{\prime}$ on $C^{\prime}$, and the proper preimage $l_{x}^{\prime} \subset \tilde{R}_{n}$ of the tangent line $l_{x}$ to $C$ at $x$ intersects $C^{\prime}$ transversally at $x^{\prime}$. Since, by assumption, on $X$ doesn't lie a 1-dimensional family of pairs of intersecting lines then the tangent line $l_{x}$ to $C$ at the general point $x \in C$ does not intersect any other tangent line to $C$.

Therefore the non-singular surface $\tilde{R}_{n}$ has a structure of a possibly nonminimal ruled surface with a general fiber $L^{\prime}:=$ the proper $\sigma$-preimage of the general tangent line $l_{x}$ to $C$. In particular $K_{\tilde{R}_{n}} \cdot L^{\prime}=-2$, and since the curve $C^{\prime}$ is a section of $\tilde{R}_{n}$ then $C^{\prime} \cdot L^{\prime}=1$.

By the definition of $C_{i}^{\prime}$ the curves $\sigma\left(C_{i}^{\prime}\right) \subset R_{o}$ are irreducible components of $\Delta$; and since by (2.2) the components of $\Delta$ can be only tangent lines to $C$ then $\sigma\left(C_{i}^{\prime}\right)$ is a tangent line to $C$. Therefore any component of $\sigma^{-1}\left(\sigma\left(C_{i}^{\prime}\right)\right)$, in particular $C_{i}^{\prime}$, will not intersect the general fiber $L^{\prime}$ of $\tilde{R}_{n}$, i.e. $C_{i}^{\prime} \cdot L^{\prime}=0$.

Moreover a contractable curve $E_{J}$ can't intersect the general fiber of $\tilde{R}_{n}$ since otherwise the point $\sigma\left(E_{J}\right) \in R_{o}$ will be a common point of a 1-dimensional family of tangent lines to $C$. The last is impossible since $g \geq 4$ and the smooth $X=$ $X_{2 g-2}$ can't contain cones-see (1.2). Therefore $E_{J} . L^{\prime}=0$ for any $j=1, \ldots, k$; and since $E$ is a sum of such $E_{J}$ then $E . L^{\prime}=0$.

Since $K_{X} \sim-H$ and $R_{o} \sim d H$ on $X$ then, by adjunction, $\left.K_{R_{o}} \sim(d-1) H\right|_{R_{o}}$. Since the hyperplane section $H$ intersects the general tangent line $l$ to $C$ at one point then $\sigma^{*}\left(\left.H\right|_{R_{o}}\right)$ is also a section of $\tilde{R}_{n}$, i.e. $\sigma^{*}\left(\left.H\right|_{R_{o}}\right) \cdot L^{\prime}=1$. Therefore

$$
\begin{aligned}
-2 & =K_{\tilde{R}_{o}} \cdot L^{\prime}=\left(\sigma^{*} K_{R_{o}}-m C^{\prime}-\Sigma_{l=1, \ldots, r} p_{i} C_{i}^{\prime}+E\right) \cdot L^{\prime} \\
& =(d-1) \sigma^{*}\left(\left.H\right|_{R_{o}}\right) \cdot L^{\prime}-m C^{\prime} \cdot L^{\prime}-\Sigma_{l=1, \ldots, r} p_{i} C_{i}^{\prime} \cdot L^{\prime}+E \cdot L^{\prime} \\
& =(d-1)-m, \text { i.e. } d=m-1 .
\end{aligned}
$$

Since $X=X_{2 g-2}$ is smooth and $g \geq 4$ then, by Lemma (A), $d>1$. Therefore $m=d+1>2$, which is impossible since $m=2$ by Lemma 2.3 . 
This contradicts the initial assumption (2.0) that the smooth $X=X_{2 g-2}, 4 \leq$ $g \leq 9$ does not contain a 1-dimensional family of conics of rank 2 . q.e.d.

\section{§3. Proof of Lemma (A)}

3.1. By [M1], [M2] any smooth prime Fano threefold $X_{2 g-2} \subset \boldsymbol{P}^{g+1}, 3 \leq$ $g \leq 10$ is a complete intersection of hypersurfaces $F_{1}, F_{2}, \ldots, F_{N}$ of degrees $d_{1}$, $d_{2}, \ldots, d_{N}$ in a homogeneous (for $g=6$ - an almost homogeneous) space $\Sigma(g)$, and:

if $g=3$ then $\Sigma(3)=\boldsymbol{P}^{4}, N=1, d_{1}=4$;

if $g=4$ then $\Sigma(4)=\boldsymbol{P}^{5}, N=2, d_{1}=2, d_{2}=3$;

if $g=5$ then $\Sigma(5)=\boldsymbol{P}^{6}, N=3, d_{1}=d_{2}=d_{3}=2$;

if $g=6$ then $\Sigma(6)=K \cdot G(2,5) \subset \boldsymbol{P}^{10}$ is a cone over the grassmannian $G(2,5)$ $\subset \boldsymbol{P}^{9}, N=4, d_{1}=d_{2}=d_{3}=1, d_{4}=2$;

if $7 \leq g \leq 10$ then $X_{2 g-2}=\Sigma(g) \cap \boldsymbol{P}^{g+1}$, where $\Sigma(7) \subset \boldsymbol{P}^{15}$ is the spinor 10fold, $\Sigma(8)=G(2,6) \subset \boldsymbol{P}^{14}, \Sigma(9) \subset \boldsymbol{P}^{13}$ is the sympletic grassmann 6-fold, and $\Sigma(10) \subset P^{13}$ is the $G_{2}$-fivefold.

3.2. To prove Lemma (A), it is enough to see that if $X=X_{2 g-2} \subset \Sigma(g)$ is a 3 -fold complete intersection as in (3.1) (assuming implicitly that such $X$ may have singularities) then $X_{2 g-2}$ can't be smooth. We shall prove this separately for any value of $g, 3 \leq g \leq 9$.

For $g=3,4,5,6,8$ we use that $\Sigma(g)$ is either a projective space or a (cone over) grassmannian, which makes it possible to compute directly that the general such $X_{2 g-2} \supset S_{2 g-2}$ must have $12-g$ singular points on the curve $C_{g}$.

For $g=7,9$ we assume that $X=X_{2 g-2} \subset S_{2 g-2}$ is smooth, and then project $X$ from a tangent line to $C_{g}$ to derive a contradiction on the base of the already known Lemma (A) for $g=5$.

3.3. Notation. Let $n \geq 1, \quad m \geq 0$ be integers, let $\boldsymbol{P}^{n+m}(z: w)=$ $\boldsymbol{P}^{n+m}\left(z_{0}: \cdots: z_{n}: w_{n+1}: \cdots: w_{n+m}\right)$ be the complex projective $(n+m)$-space, and let $F(z: w)=F\left(z_{0}: \cdots: z_{n}: w_{n+1}: \cdots: w_{n+m}\right)$ be a homogeneous form. Denote by $\nabla_{z} F=\left(\partial F / \partial z_{0}, \ldots, \partial F / \partial z_{n}\right)$ the gradient vector of $F$ with respect to $(z)=$ $\left(z_{0}: \cdots: z_{n}\right)$.

Let $F_{1}(z: w), \ldots, F_{k}(z: w)(k \geq 1)$ be homogeneous forms. Denote by:

$\left(F_{1}, \ldots, F_{k}\right) \subset \boldsymbol{C}[z: w]=\boldsymbol{C}\left[z_{0}: \cdots: z_{n}: w_{n+1}: \cdots: w_{n+m}\right]$ - the homogeneous ideal generated by $F_{1}, \ldots, F_{k}$;

$V\left(F_{1}, \ldots, F_{k}\right)$ - the projective variety defined by $F_{1}, \ldots, F_{k}$;

$\left.J_{z}\right|_{(a: b)}=\left.J_{z}\left(F_{1}, \ldots, F_{k}\right)\right|_{(a: b)}=\left.\left[\nabla_{z} F_{1} ; \ldots ; \nabla_{z} F_{k}\right]\right|_{(a: b)}$-the Jacobian matrix $J_{z}$ of partial derivatives of $F_{1}, \ldots, F_{k}$ with respect to $(z)=\left(z_{0}, \ldots, z_{n}\right)$, computed at the point $(a: b) \in \boldsymbol{P}^{n+m}(z: w)$, (where $\nabla_{z} F_{i}$ are regarded as rows of $\left.J_{z}\right)$.

Let e.g. $m=0$, let $X=V\left(F_{1}, \ldots, F_{k}\right) \subset \boldsymbol{P}^{n}\left(z_{0}: \cdots: z_{n}\right)$, and let $\operatorname{dim} X=d$. Then $\operatorname{dim} T_{x} X \geq d$ for any $x \in X$, where $T_{x} X$ is the tangent space to $X$ at $x$; and the point $x \in X$ is singular if $\operatorname{dim} T_{x} X>d$ (see e.g. Chapter 2, $\S 1.4$ in [Sh]). 
Equivalently $x \in X$ is singular if rank $\left.J_{z}\right|_{x}<n-d$. The subset $\operatorname{Sing} X=\{x \in X$ : rank $\left.\left.J_{z}\right|_{x}<n-d\right\} \subset X$ of all the singular points of $X$ is a proper closed subset of the projective algebraic variety $X$, defined on $X$ by vanishing of all the $(n-d) \times$ $(n-d)$ minors of $J_{z}$.

3.4. Proof of Lemma (A) for $g=3$. The tangent scroll to the twisted cubic $C_{3}:\left(x_{0}: x_{1}: x_{2}: x_{3}\right)=\vec{s}:=\left(s_{0}^{3}: s_{0}^{2} s_{1}: s_{0} s_{1}^{2}: s_{1}^{3}\right)$ is the quartic surface $S_{4}=V(f)$ $\subset \boldsymbol{P}^{3}(x)$ where $f(x)=3 x_{1}^{2} x_{2}^{2}+6 x_{0} x_{1} x_{2} x_{3}-4 x_{1}^{3} x_{3}-x_{0}^{2} x_{3}^{2}-4 x_{0}^{2} x_{2}^{3}$. The surface $S_{4}$ is singular along $C_{3}$ since the gradient vector $\left.\nabla_{x} f\right|_{\vec{s}}=0$ for any $\vec{s} \in C_{3}$.

Let the quartic threefold $X_{4} \subset \boldsymbol{P}^{4}(x: u)$ be such that $S_{4}=X_{4} \cap \boldsymbol{P}^{3}(x)$, and let $X_{4}=V(F) \subset \boldsymbol{P}^{4}(x: u)$ where $F(x: u)=\Sigma_{0 \leq k \leq 4} f_{i}(x) u^{4-t}$. Therefore $f_{4} \in(f)$, i.e. $f_{4}=c f$ for some constant $c \in C$.

Let $x \in X_{4}$. Then $x \in \operatorname{Sing} X_{4}$ iff $\nabla_{x, u} F_{x}=0$. Let $s=\left(s_{0}: s_{1}\right) \in \boldsymbol{P}^{1}$. Then $(\vec{s}: 0) \in \operatorname{Sing} X_{4}$ iff $0=\left.\nabla_{x, u} F\right|_{(\vec{s}: 0)}=\left.\left(\nabla_{x} F, \partial F / \partial u\right)\right|_{(\vec{s}: 0)}=\left(\left.\nabla_{x} f\right|_{\vec{s}}, f_{3}(\vec{s})\right)=\left(0, f_{3}(\vec{s})\right)$.

Therefore either $f_{3}(\vec{s}) \equiv 0$ (i.e. $f_{3}(\vec{s})=0$ for any $\left.s=\left(s_{0}: s_{1}\right)\right)$, and then $X_{4}$ is singular along $\overline{C_{4}}$, or $f_{s}(\vec{s}) \not \equiv 0$, and then $(\vec{s}: 0) \in \operatorname{Sing} X_{4}$ iff $s=\left(s_{0}: s_{1}\right)$ is a zero of the (non-vanishing) homogeneous form $F_{9}(s)=f_{3}(\vec{s})=f_{3}\left(s_{0}^{3}: s_{0}^{2} s_{1}: s_{0} s_{1}^{2}: s_{1}^{3}\right)$ of degree 9.

In addition, for the general $f_{3}(x)$ all the zeros of $F_{9}(s)=f_{3}(\vec{s})$ are simple, i.e. different from each other. Therefore the general $X_{4} \supset S_{4}$ has $9=12-g\left(X_{4}\right)$ singular points on $C_{3}$. In coordinates as above, these singular points of $X_{4}$ are the images of the 9 zeros of $F_{9}(s)$ under the Veronese map $v_{3}: \boldsymbol{P}^{1} \rightarrow C_{3} \subset X_{4}$, $v_{3}: s=\left(s_{0}: s_{1}\right) \mapsto(\vec{s}: 0)$.

3.5. Proof of Lemma (A) for $g=4$. The tangent scroll to the rational normal quartic $C_{4}:\left(x_{0}: x_{1}: \cdots: x_{4}\right)=\vec{s}:=\left(s_{0}^{4}: s_{0}^{3} s_{1}: \cdots: s_{1}^{4}\right)$ is a complete intersection $S_{6}=V(q, f) \subset \boldsymbol{P}^{4}(x)$ where $q(x)=3 x_{2}^{2}-4 x_{1} x_{3}+x_{0} x_{4}$ and $f(x)=$ $x_{2}^{3}-2 x_{0} x_{3}^{2}-2 x_{1}^{2} x_{4}+3 x_{0} x_{2} x_{4}$. The surface $S_{6}$ is singular along $C_{4}$ since the gradients of $q$ and $f$ are linearly dependent along $C_{4}$; more precisely $\left.\nabla_{x} f\right|_{\vec{s}}=$ $\left.s_{0}^{2} s_{1}^{2} \nabla_{x} q\right|_{\vec{s}}$ for any $\vec{s} \in C_{4}$.

Let $X_{6}=V(Q, F) \subset \boldsymbol{P}^{5}(x: u)$ be a complete intersection of the quadric $Q(x: z)=\Sigma_{0 \leq k \leq 2} q_{k}(x) u^{2-k}=0$ and the cubic $F(x: z)=\Sigma_{0 \leq l \leq 3} q_{l}(x) u^{3-l}=0$, and let $S_{6}=X_{6} \cap \boldsymbol{P}^{4}(x)$. In particular, $\left(q_{2}, f_{3}\right) \subset(q, f)$ as homogeneous ideals in $\boldsymbol{C}[x]=\boldsymbol{C}\left[x_{0}: \cdots: x_{4}\right]$.

For the fixed $X_{6}=V(Q, F)$ the generators $Q, F$ of the homogeneous ideal $(Q, F)$ can be replaced by $c^{\prime} Q$ and $c^{\prime \prime} F+L(x: u) Q$, for any pair of nonzero constants $c^{\prime}$ and $c^{\prime \prime}$, and for any linear form $L(x: u)$. Now, $\left(q_{2}, f_{3}\right) \subset(q, f)$ yields that one can choose $Q$ and $F$ such that $q_{2}=\varepsilon^{\prime} q$ and $f_{3}=\varepsilon^{\prime \prime} f$, where $\varepsilon^{\prime}, \varepsilon^{\prime \prime}$ are either 0 or 1 .

Consider the general case $\varepsilon^{\prime}=\varepsilon^{\prime \prime}=1$; the study in the degenerate case $\varepsilon^{\prime} \cdot \varepsilon^{\prime \prime}$ $=0$ is similar. The subscheme $\operatorname{Sing} X_{6}=\operatorname{Sing} V(Q, F)$ is defined by $\operatorname{rank}\left[\nabla_{x, u} Q\right.$; $\left.\nabla_{x, u} F\right] \leq 1$. By the choice of $Q$ and $F,\left.\nabla_{x, u} Q\right|_{(\vec{s}: 0)}=\left(\left.\nabla_{x} q_{2}\right|_{\vec{s}}, q_{1}(\vec{s})\right)$ and $\left.\nabla_{x, u} F\right|_{(\vec{s}: 0)}$ $=\left(\left.\nabla_{x} f_{3}\right|_{\vec{s}}, f_{2}(\vec{s})\right)$, where $1 . q_{2}=q$ and $1 . f_{3}=f$. Just as in case $g=3$, the last 
together with the identity $\left.\nabla_{x} f\right|_{\vec{s}}-\left.s_{0}^{2} s_{1}^{2} \nabla_{x} q\right|_{\vec{s}} \equiv 0$ imply that $(\vec{s}: 0) \in \operatorname{Sing} X_{6}$ iff $F_{8}(s):=f_{2}(\vec{s})-s_{0}^{2} s_{1}^{2} \cdot q_{1}(\vec{s})=0$, where $\vec{s}=\left(s_{0}^{4}: s_{0}^{3} s_{1}: \cdots: s_{1}^{4}\right)$.

The Veronese map $v_{4}: \boldsymbol{P}^{1} \rightarrow C_{4} \subset X_{6}, v_{4}: s=\left(s_{0}: s_{1}\right) \mapsto(\vec{s}: 0)$ states an isomorphism between $\boldsymbol{P}^{1}$ and $C_{4}$. Therefore either $F_{8}(s) \equiv 0$, and then $X_{6}$ is singular along $C_{4}$, or $F_{8}(s) \not \equiv 0$, and then the singular points of $X_{6}$ on $C_{4}$ are the $v_{4}$-images of the zeros of the homogeneous form $F_{8}(s)$ of degree 8 . As in case $g=3$, for the general $f_{2}(x), q_{1}(x)$ the form $F_{8}(s)$ has only simple zeros. Therefore the general $X_{6} \supset S_{6}$ has $8=12-g\left(X_{6}\right)$ singular points on $C_{4}$.

3.6. Proof of Lemma (A) for $g=5$. The tangent scroll to the rational normal quintic $C_{5}: x_{l}=s_{0}^{5-l} s_{1}^{l}, \quad 0 \leq i \leq 5$ is a complete intersection $S_{8}=$ $V\left(q^{\prime}, q^{\prime \prime}, q^{\prime \prime \prime}\right) \subset \boldsymbol{P}^{5}(x)$ where $q^{\prime}(x)=4 x_{1} x_{3}-3 x_{2}^{2}-x_{0} x_{4}, q^{\prime \prime}(x)=3 x_{1} x_{4}-2 x_{2} x_{3}-$ $x_{0} x_{5}$ and $q^{\prime \prime \prime}(x)=x_{1} x_{5}-4 x_{2} x_{4}+3 x_{3}^{2}$. The surface $S_{8}$ is singular along $C_{4}$ since the gradients of $q^{\prime}, q^{\prime \prime}$ and $q^{\prime \prime \prime}$ are linearly dependent along $C_{5}$. More precisely

$$
\left.s_{1}^{2} \nabla_{x} q^{\prime}\right|_{\vec{s}}-\left.s_{0} s_{1} \nabla_{x} q^{\prime \prime}\right|_{\vec{s}}-\left.s_{0}^{2} \nabla_{x} q^{\prime \prime \prime}\right|_{\vec{s}}=0 \text { for any } \vec{s} \in C_{5} .
$$

Let $X_{8}=V\left(Q^{\prime}, Q^{\prime \prime}, Q^{\prime \prime \prime}\right) \subset \boldsymbol{P}^{6}(x: u)$ be a complete intersection of the quadrics $Q^{l}(x: z)=\Sigma_{0 \leq k \leq 2} q_{k}^{l}(x) u^{2-k}\left(i={ }^{\prime},{ }^{\prime \prime},{ }^{\prime \prime \prime}\right)$, and such that $S_{8}=X_{8} \cap \boldsymbol{P}^{5}(x)$. In particular $\left(q_{1}^{\prime}, q_{1}^{\prime \prime}, q_{1}^{\prime \prime \prime}\right) \subset\left(q^{\prime}, q^{\prime \prime}, q^{\prime \prime \prime}\right)$ as homogeneous ideals in $\boldsymbol{C}[x]=\boldsymbol{C}\left[x_{0}: \cdots: x_{4}\right]$. Therefore $q_{1}^{\prime}, q_{1}^{\prime \prime}$ and $q_{1}^{\prime \prime \prime}$ are linear combinations of $q^{\prime}, q^{\prime \prime}$ and $q^{\prime \prime \prime}$. Since the ideal $\left(Q^{\prime}, Q^{\prime \prime}, Q^{\prime \prime}\right)$ of $X_{8}$ is generated also by any $G L(3)$-transform of the triple $\left(Q^{\prime}, Q^{\prime \prime}, Q^{\prime \prime \prime}\right)$, we may assume that $q_{2}^{\prime}=\varepsilon^{\prime} q^{\prime}, q_{2}^{\prime \prime}=\varepsilon^{\prime \prime} q^{\prime \prime}$ and $q_{2}^{\prime \prime \prime}=\varepsilon^{\prime \prime \prime} q^{\prime \prime \prime}$, where $\varepsilon^{\prime}, \varepsilon^{\prime \prime}$ and $\varepsilon^{\prime \prime \prime}$ are 0 or 1 . The subscheme $\left.\left(\operatorname{Sing} X_{8}\right)\right|_{C_{5}} \subset \boldsymbol{P}^{1}$ is defined by

$$
\begin{aligned}
2 & \geq\left.\operatorname{rank}\left[\nabla_{x, u} Q^{\prime} ; \nabla_{x, u} Q^{\prime \prime} ; \nabla_{x, u} Q^{\prime \prime \prime}\right]\right|_{(\vec{s}: 0)} \\
& =\operatorname{rank}\left[\left(\left.\nabla_{x} \varepsilon^{\prime} q^{\prime}\right|_{\vec{s}}, q_{1}^{\prime}(\vec{s})\right) ;\left(\left.\nabla_{x} \varepsilon^{\prime \prime} q^{\prime \prime}\right|_{\vec{s}}, q_{1}^{\prime \prime}(\vec{s})\right) ;\left(\left.\nabla_{x} \varepsilon^{\prime \prime \prime} q^{\prime \prime \prime}\right|_{\vec{s}}, q_{1}^{\prime \prime \prime}(\vec{s})\right)\right] .
\end{aligned}
$$

Let $F_{7}(s)=\varepsilon^{\prime} s_{1}^{2} q_{1}^{\prime}(\vec{s})-\varepsilon^{\prime \prime} s_{0} s_{1} q_{1}^{\prime \prime}(\vec{s})-\varepsilon^{\prime \prime \prime} s_{0}^{2} q_{1}^{\prime \prime \prime}(\vec{s})$.

The Veronese map $v_{5}: \boldsymbol{P}^{1} \rightarrow C_{5} \subset X_{8}, v_{5}: s=\left(s_{0}: s_{1}\right) \mapsto(\vec{s}: 0)$ states an isomorphism between $\boldsymbol{P}^{1}$ and $C_{5}$. Just as in (3.5), (*) and (**) imply that either $F_{7}(s) \equiv 0$, and then $X_{8}$ is singular along $C_{5}$, or $F_{7}(s) \not \equiv 0$, and then the singular points of $X_{6}$ on $C_{5}$ are the $v_{5}$-images of the zeros of the homogeneous form $F_{7}(s)$ of degree 7. Moreover, for the general linear forms $q_{1}^{\prime}(x), q_{1}^{\prime \prime}(x), q_{1}^{\prime \prime \prime}(x)$ the form $F_{7}(s)$ has only simple zeros. Therefore the general $X_{8} \supset S_{8}$ has $7=12-g\left(X_{8}\right)$ singular points on $C_{5}$.

$$
\text { Lemma (A) for } g=6,8 \text {. }
$$

Lemma 3.7. Let $n \geq 3$, and let $G=G(1: n)=G(2, n+1) \subset \boldsymbol{P}^{n(n+1) / 2-1}$ be the grassmannian of lines in $\boldsymbol{P}^{n}=\boldsymbol{P}\left(\boldsymbol{C}^{n+1}\right)$. Let $C \subset G \subset \boldsymbol{P}^{n(n+1) / 2-1}$ be a smooth irreducible curve such that $\operatorname{dim} \operatorname{Span}(C) \geq 3$. Let the surface $\operatorname{Gr}(C) \subset \boldsymbol{P}^{n}$ be the union of lines $l \subset \boldsymbol{P}^{n}$ such that $l \in C \subset G=G(1: n)$, and let $S_{C} \subset \operatorname{Span}(C) \subset$ $\boldsymbol{P}^{n(n+1) / 2-1}$ be the surface swept out by the tangent lines to $C$ (or the tangent scroll to $C$-see above). 
Then the tangent scroll $S_{C}$ to $C$ lies on $G=G(1: n)$ iff

either all the lines $l \in C$ have a common point, i.e. $\operatorname{Gr}(C)$ is a cone,

or there exists an trreducible curve $Z \subset \boldsymbol{P}^{n}$ such that all the lines $l \in C$ are tangent lines to $Z$, i.e. $\operatorname{Gr}(C)$ is the tangent scroll to $Z$.

Proof. For $n=3$ this result can be found in [AS]. For $n>3$ one can apply induction, using the fact that projection from a point in $\boldsymbol{P}^{n}$ induces a projection from $G(2, n+1)$ onto $G(2, n)$.

q.e.d.

Lemma 3.8. Let $C_{g} \subset G(2, g / 2+2)=G\left(1: \boldsymbol{P}^{g / 2+1}\right)(g=6,8)$ be a rational normal curve such that the tangent scroll $S_{2 g-2}$ to $C_{g}$ is contained in $G(2, g / 2+2)$. Then:

(i). If $g=6$ then the lines $l \subset \boldsymbol{P}^{4}, l \in C_{6}$ sweep out the tangent scroll to a rational normal curve $C_{4} \subset \boldsymbol{P}^{4}$.

(ii). If $g=8$, and if there exists a 3-fold linear section $X_{14}=G(2,6) \cap \boldsymbol{P}^{9}$ such that $X_{14} \supset S_{14}$, then the lines $\left\{l \subset \boldsymbol{P}^{5}: l \in C_{8}\right\}$ sweep out the tangent scroll to a rational normal curve $C_{5} \subset \boldsymbol{P}^{5}$.

Proof. Let $g=6,8$, let the lines $l \in C_{g}$ sweep out a cone $\operatorname{Gr}\left(C_{g}\right) \subset \boldsymbol{P}^{g / 2+1}$ see Lemma 3.7, and let $x$ be the vertex of $\operatorname{Gr}\left(C_{g}\right)$. Then $C_{g}$ is contained in the Schubert $g / 2$-space $\boldsymbol{P}_{x}^{g / 2}=\sigma_{g / 2,0}(x)=\left\{l \subset \boldsymbol{P}^{g / 2+1}: x \in l\right\}$. Since $C_{g}$ is projectively normal it must span a $g$-space. Therefore $g \leq g / 2+1$ which contradicts $g=6,8$. Therefore, by Lemma 3.7, the lines $l \in C_{g}$ must sweep out the tangent scroll to a rational curve $C_{d} \subset \boldsymbol{P}^{g / 2+1}$.

Let $g=6$, and let $C_{d} \subset \boldsymbol{P}^{3} \subset \boldsymbol{P}^{4}$. Then $C_{6} \subset G\left(1: \boldsymbol{P}^{3}\right)=\sigma_{11}\left(\boldsymbol{P}^{3}\right) \subset G\left(1: \boldsymbol{P}^{4}\right)$. Therefore $6=\operatorname{dim}\left(\operatorname{Span} C_{6}\right) \leq \operatorname{dim}\left(\operatorname{Span} G\left(1: \boldsymbol{P}^{3}\right)\right)=5$-contradiction. Therefore $d \geq 4$ since $C_{d}$ must span $\boldsymbol{P}^{4}$, and now it is easy to see that the rational normal curve $C_{6}$ is the curve of tangent lines to $C_{d}$ iff $d=4$.

Let $g=8$, and let $C_{d} \subset \boldsymbol{P}^{4} \subset \boldsymbol{P}^{5}$. Then $C_{8} \subset G\left(1: \boldsymbol{P}^{4}\right)=\sigma_{11}\left(\boldsymbol{P}^{4}\right) \subset$ $G\left(1: \boldsymbol{P}^{5}\right)$. Let $\boldsymbol{P}_{o}^{9}=\operatorname{Span} G\left(1: \boldsymbol{P}^{4}\right)$. Then $\boldsymbol{P}^{8}=\operatorname{Span} C_{8} \subset \boldsymbol{P}_{o}^{9}$. By condition $C_{8} \subset X_{14}=G\left(1: \boldsymbol{P}^{5}\right) \cap \boldsymbol{P}^{9}$. Therefore $\boldsymbol{P}^{8} \subset \boldsymbol{P}^{9} \cap \boldsymbol{P}_{o}^{9}$ and $X_{14} \supset Z_{o}:=G\left(1: \boldsymbol{P}^{4}\right)$ $\cap \boldsymbol{P}^{8}$. Since $\boldsymbol{P}^{8} \subset \boldsymbol{P}_{o}^{9}=\operatorname{Span} G\left(1: \boldsymbol{P}^{4}\right), Z_{o}$ is at least a hyperplane section of the 6-dimensional grassmannian $G\left(1: \boldsymbol{P}^{4}\right)$. This contradicts $X_{14} \supset Z_{o}$ and $\operatorname{dim} X_{14}$ =3. Therefore $d \geq 5$ since $\operatorname{Span} C_{d}=\boldsymbol{P}^{5}$, and now it is easy to see that the rational normal curve $C_{8}$ is the curve of tangent lines to $C_{d}$ iff $d=5$. q.e.d.

Proof of Lemma (A) for $g=6$.

3.9. By (3.1) any smooth prime $X_{10}$ is a complete intersection of three hyperplanes and a quadric in the cone $K . G(2,5) \subset \boldsymbol{P}^{10}$. Let $o$ be the vertex of the cone $K . G(2,5) \subset \boldsymbol{P}^{10}$, and let $X \subset K . G(2,5)$, be as in (3.1). There are two kinds of such threefolds $X_{10}$ (see [I1], $[\mathrm{Gu}]$ ):

(i). $g=6$-first kind: $o \notin \operatorname{Span}(X)$, and then the projection $p_{o}$ from $o$ sends $X$ isomorphically to $X_{10}=G(2,5) \cap \boldsymbol{P}^{7} \cap Q$, where $G(2,5) \subset \boldsymbol{P}^{9}$ by the Plücker embedding, $\boldsymbol{P}^{7} \subset \boldsymbol{P}^{9}$ and $Q$ is a quadric. 
(ii). $g=6$ - second kind: $o \in \operatorname{Span}(X)$, and then $\pi=\left.p_{o}\right|_{X}: X=X_{10}^{\prime} \rightarrow Y_{5}$ is a double covering of a threefold $Y_{5}=G(2,5) \cap \boldsymbol{P}^{6}$. In particular, if $X_{10}^{\prime}$ is smooth then the intersection $Y_{5}=G(2,5) \cap \boldsymbol{P}^{6}$ is smooth.

$$
g=6 \text { (first kind) }
$$

3.10. Let $X_{10}=G(2,5) \cap \boldsymbol{P}^{7} \cap Q$ be a (possibly singular) complete intersection as in (3.9)(i), and assume that $X_{10}$ contains the tangent scroll $S_{10}$ to a rational normal curve $C_{6}$ of degree 6 . By Lemma 3.8(i) the points of $C_{6}$ are the Plücker coordinates $x_{i j}(s)$ of the tangent lines to a rational normal quartic $C_{4}=x_{\imath}=s^{l}, 0 \leq i \leq 4$, i.e.

$$
\left(x_{i j}(s)\right)=\left(\begin{array}{ccccc}
0 & 1 & 2 s & 3 s^{2} & 4 s^{3} \\
\cdots & 0 & s^{2} & 2 s^{3} & 3 s^{4} \\
\cdots & \cdots & 0 & s^{4} & 2 s^{5} \\
\cdots & \ldots & \cdots & 0 & s^{6} \\
\cdots & \ldots & \ldots & \ldots & 0
\end{array}\right)
$$

Therefore the subspace $\boldsymbol{P}^{6}=\operatorname{Span}\left(C_{6}\right) \subset \boldsymbol{P}^{9}$ is defined by $H_{0}=H_{1}=H_{2}=0$ where $H_{0}=x_{03}-3 x_{12}, H_{1}=x_{04}-2 x_{13}$ and $H_{2}=x_{14}-3 x_{23}$.

3.11. Introduce in $\boldsymbol{P}^{6}$ the coordinates $(v)=\left(v_{0}: \cdots: v_{6}\right)=\left(x_{01}: x_{02}: x_{12}:\right.$ $\left.x_{13}: x_{23}: x_{24}: x_{34}\right)$, and let $I_{S_{10}} \subset \boldsymbol{C}[v]:=\boldsymbol{C}\left[v_{0}, \ldots, v_{6}\right]$ be the homogeneous ideal of the tangent scroll $S_{10} \subset \boldsymbol{P}^{6}$ to $C_{6}$. Let $P f_{k}=x_{a b} x_{c d}-x_{a c} x_{b d}+x_{a d} x_{b c}, 0 \leq$ $a<b<c<d \leq 4: a, b, c, d \neq k(k \in\{0,1,2,3,4\})$ be the 5 Plücker quadrics in the coordinates $x_{j k}$. In coordinates $(v)$ of $\boldsymbol{P}^{6}=\boldsymbol{P}^{6}(v)$ the restrictions $q_{k}$ of $\boldsymbol{P} f_{k}$ to $P^{6}$ are

$$
\begin{gathered}
q_{0}(v)=v_{2} v_{6}-v_{3} v_{5}+3 v_{4}^{2}, \quad q_{1}(v)=v_{1} v_{6}-3 v_{2} v_{5}+2 v_{3} v_{4} \\
q_{2}(v)=v_{0} v_{6}-9 v_{2} v_{4}+2 v_{3}^{2}, \quad q_{3}(v)=v_{0} v_{5}-3 v_{1} v_{4}+2 v_{2} v_{3} \\
q_{4}(v)=v_{0} v_{4}-v_{1} v_{3}+3 v_{2}^{2} .
\end{gathered}
$$

In the open subset $U_{0}=\left\{v_{0}=1\right\} \subset \boldsymbol{P}^{6}(v)$ the curve $C_{6}$ is parameterized by $C_{6}=\left\{(v)=\vec{s}:=\left(1,2 s, s^{2}, 2 s^{3}, s^{4}, 2 s^{5}, s^{6}\right)\right\}$.

Now, it is easy to see that the quadric $q(v)=5 v_{2} v_{4}-2 v_{1} v_{5}+3 v_{0} v_{6}$ vanishes at the points of the tangent scroll $S_{10}$ to $C_{6}$, and the homogeneous ideal $I_{S_{10}}=$ $\left(q_{0}, \ldots, q_{4}, q\right) \subset \boldsymbol{C}[v]$.

Let $J_{v}=J_{v}\left(q_{0}, \ldots, q_{4}, q\right)$ be the Jacobian matrix of $\left(q_{0}, \ldots, q_{4}, q\right)$ with respect to $v=\left(v_{1}, \ldots, v_{6}\right)$. The surface $S_{10} \subset V_{5}$ is singular along $C_{6}$ since $\left.\operatorname{rank} J_{v}\right|_{\vec{s}}<$ $4=\operatorname{codim}_{P^{6}} S_{10}$ for any $\vec{s} \in C_{6}$. For the special choice $(v)$ of the coordinates this can be verified directly:

$$
\begin{aligned}
\left.s^{-2} \nabla_{v} q_{0}\right|_{\vec{s}}=\left(0, s^{4},-2 s^{3}, 6 s^{2},-2 s, 1\right), & \left.s^{-1} \nabla_{v} q_{1}\right|_{\vec{s}}=\left(s^{5},-6 s^{4}, 2 s^{3}, 4 s^{2},-3 s, 2\right), \\
\left.\nabla_{v} q_{2}\right|_{\vec{s}}=\left(0,-9 s^{4}, 8 s^{3},-9 s^{2}, 0,1\right), & \left.s \nabla_{v} q_{3}\right|_{\vec{s}}=\left(-3 s^{5}, 4 s^{4}, 2 s^{3},-6 s^{2}, s, 0\right), \\
\left.s^{2} \nabla_{v} q_{4}\right|_{\vec{s}}=\left(-2 s^{3}, 6 s^{4},-2 s^{3}, s^{2}, 0,0\right), & \text { and }\left.\nabla_{v} q\right|_{\vec{s}}=\left(-4 s^{5}, 5 s^{4}, 0,5 s^{2},-4 s, 3\right) .
\end{aligned}
$$


(*). Therefore rank $\left.J_{v}\right|_{\vec{s}}=4$ for any $\vec{s} \in C_{6}$, and the linear 4-space of linear equations between the gradients $\left.\nabla_{v} q_{0}\right|_{\vec{s}}, \ldots,\left.\nabla_{v} q_{4}\right|_{\vec{s}}$ and $\left.\nabla_{v} q\right|_{\vec{s}}$ is spanned on the Pfaff-equation $\left.s^{-2} \nabla_{v} q_{0}\right|_{\vec{s}}+\left.s^{-1} \nabla_{v} q_{1}\right|_{\vec{s}}+\left.\nabla_{v} q_{2}\right|_{\vec{s}}+\left.s \nabla_{v} q_{3}\right|_{\vec{s}}+\left.s^{2} \nabla_{v} q_{4}\right|_{\vec{s}}=0$ and the 3space of equations $\left.\nabla_{v} q\right|_{\vec{s}}=a_{0} .\left.s^{-2} \nabla_{v} q_{0}\right|_{\vec{s}}+a_{1} .\left.s^{-1} \nabla_{v} q_{1}\right|_{\vec{s}}+a_{2} .\left.\nabla_{v} q_{2}\right|_{\vec{s}}+a_{3} .\left.s \nabla_{v} q_{3}\right|_{\vec{s}}+$ $a_{4} .\left.s^{2} \nabla_{v} q_{4}\right|_{\vec{s}}=0$ where:

$$
(* *) . \quad a_{0}+4 a_{3}+3 a_{4}=8, a_{1}-3 a_{3}-2 a_{4}=-4, a_{2}+2 a_{3}+a_{4}=3 .
$$

3.12. In the dual space $\check{\boldsymbol{P}}^{9}$, let $\check{\boldsymbol{G}}=\check{G}(2,5) \subset \check{\boldsymbol{P}}^{9}$ be the grassmannian of hyperplane equations represented by the skew-symmetric $5 \times 5$ matrices of rank 2 . It is easy to see that the plane $\Pi=\left\langle H_{0}, H_{1}, H_{2}\right\rangle \subset \check{\boldsymbol{P}}^{9}$ of hyperplane equations of $\boldsymbol{P}^{6}=\operatorname{Span} C_{6}$ does not intersect $\check{G}$.

Let $\Lambda \subset \Pi$ be any line in $\Pi$. In $\boldsymbol{P}^{9}$, the line $\Lambda$ defines, by duality, the subspace $\boldsymbol{P}^{7}(\Lambda) \supset \boldsymbol{P}^{6}=\operatorname{Span} C_{6}$. It is easy to see that the fourfold $W(\Lambda)=$ $G \cap \boldsymbol{P}^{7}(\Lambda)$, where $G=G(2,5)$, is smooth. In fact, $W(\Lambda)$ will be smooth iff the line $\Lambda$ does not intersect $\check{G}$. The last is true since $\Lambda \subset \Pi$ and $\Pi \cap \check{G}=\emptyset$. Therefore any $X_{10} \supset S_{10}$ is a quadratic section of the smooth 4-fold $W=W(\Lambda)$.

3.13. Let $X_{10}=G(2,5) \cap \boldsymbol{P}^{7} \cap Q \supset S_{10}$, where $Q$ is a quadric. We shall show that the singularities of $X_{10}$ on $C_{6}$ are the zeros of a homogeneous form of degree 6 on $C_{6} \cong \boldsymbol{P}^{1}$. To simplify the notation, we shall show this for one special choice of the line $\Lambda \subset \Pi$ (see (3.12)); the check for any other $\Lambda \subset \Pi$ is similar.

Let $\Lambda=\left\{H_{2}=0\right\} \subset \Pi$. Then, in coordinates $(v)$ and $u=x_{14}-3 x_{23}$ in $\boldsymbol{P}^{7}(\Lambda)$, the subspace $\boldsymbol{P}^{6}(x)=(u=0)$. Therefore any quadric $Q \subset \boldsymbol{P}^{7}(\Lambda)$, such that $Q \cap X_{10}=S_{10}$, can be written in the form $Q=Q(v, u)=c u^{2}+L(v) u+q(v)$ where $c \in C$ and $L$ is a linear form of $(v)$.

Let $Q_{k}(v, u)(k=0,1, \ldots, 4)$ be the restriction of the Pfaff quadric $P f_{k}$ on $\boldsymbol{P}^{7}(v, u)$, and let $J_{v, u}=\left[\nabla_{v, u} Q_{0} ; \ldots ; \nabla_{v, u} Q_{4} ; \nabla_{v, u} Q\right]$ be the Jacobian matrix of $\left(Q_{0}, \ldots, Q_{4}, Q\right)$.

The singularities of $X_{10}$ on $C_{6}$ are the points $(\vec{s}: 0) \in \boldsymbol{P}^{7}$ for which $\left.\operatorname{rank} J_{v, u}\right|_{(\vec{s}: 0)}<4=\operatorname{codim}_{\boldsymbol{P}^{7}} X_{10}$.

Let $l_{i}(\vec{s})=\partial Q_{i} /\left.\partial u\right|_{\vec{s}}, i=0, \ldots, 4$. The rows of $\left.J_{v, u}\right|_{(\vec{s}, 0)}$ are $\left.\nabla_{u, v} Q_{i}\right|_{(\vec{s}: 0)}=$ $\left(\left.\nabla_{u} q_{i}\right|_{\vec{s}}, l_{i}(\vec{s})\right), i=0, \ldots, 4$ and $\left.\nabla_{u, v} Q\right|_{(\vec{s}: 0)}=\left(\left.\nabla_{u} q\right|_{\vec{s}}, L(\vec{s})\right)$. For the special choice of the line $\Lambda \subset \Pi$, the linear forms $l_{i}=l_{i}(\vec{s})$ are $\left(l_{0}, l_{1}, l_{2}, l_{3}, l_{4}\right)=\left(s^{4}, 0,-3 s^{2}\right.$, $-2 s, 0)$.

Therefore, in view of $(*), X_{10}$ will have a singularity at $(\vec{s}, 0) \in C_{6}$ if there exist constants $a_{0}, \ldots, a_{4}$ satisfying $(* *)$ and such that $L(\vec{s})=\left(a_{0}-3 a_{2}-2 a_{3}\right) s^{2}$ (here $\vec{s}=\left(1,2 s, s^{2}, 2 s^{3}, s^{4}, 2 s^{5}, s^{6}\right)$-see above).

By $(* *) \quad a_{0}-3 a_{2}-2 a_{3}=-1$. Therefore, in homogeneous coordinates $\left(s_{0}: s_{1}\right), s=s_{1} / s_{0}$, the variety $X_{10}$ will be singular at the point $((\vec{s}): 0) \in C_{6}$ iff $F_{6}\left(s_{0}: s_{1}\right):=L\left(s_{0}^{6}: 2 s_{0}^{5} s_{1}: s_{0}^{4} s_{1}^{2}: 2 s_{0}^{3} s_{1}^{3}: s_{0}^{2} s_{1}^{4}: 2 s_{0} s_{1}^{5}: s_{1}^{6}\right)+s_{0}^{4} s_{1}^{2}=0$.

The Veronese map $v_{6}: \boldsymbol{P}^{1} \rightarrow C_{6} \subset X_{10}, v_{6}:\left(s_{0}: s_{1}\right) \mapsto(\vec{s}: 0)=\left(s_{0}^{6}: 2 s_{0}^{5} s_{1}:\right.$ $\left.s_{0}^{4} s_{1}^{2}: 2 s_{0}^{3} s_{1}^{3}: s_{0}^{2} s_{1}^{4}: 2 s_{0} s_{1}^{5}: s_{1}^{6}: 0\right)$, states an isomorphism between $\boldsymbol{P}^{1}$ and $C_{6}$. 
Therefore either $F_{6}\left(s_{0}: s_{1}\right) \equiv 0$, and then $X_{10}$ is singular along $C_{6}$, or $F_{6}\left(s_{0}: s_{1}\right) \not \equiv$ 0 , and then the singular points of $X_{10}$ on $C_{6}$ are the $v_{6}$-images of the zeros of the homogeneous form $F_{6}\left(s_{0}: s_{1}\right)$ of degree 6 .

The choice of an arbitrary line $\Lambda \subset \Pi$ will only change the homogeneous sextic forms defined by $l_{i}(\vec{s}), i=0, \ldots, 4$.

q.e.d.

$$
g=6 \text { (second kind) }
$$

3.14. Let $\pi: X=X_{10}^{\prime} \rightarrow Y_{5}, X \subset \boldsymbol{P}^{7}$, be a double covering of the Del Pezzo threefold $Y_{5}=G(2,5) \cap \boldsymbol{P}^{6}$ branched along the quadratic section $B \subset Y_{5}$. Below we shall identify the branch locus $B \subset Y_{5}$ and the ramification divisor $R \subset X_{10}^{\prime}$, $R \cong B$.

3.15. Assume that $X$ contains the tangent scroll $S=S_{10}$ to the rational normal sextic $C=C_{6}$, and let $l \subset S$ be a general tangent line to $C$. Then $N_{l / X} \cong \mathcal{O}(1) \oplus \mathcal{O}(-2) \quad($ see $(1.2))$, and $\pi(l) \subset Y_{5}$ also is a line. If $N_{\pi(l) / Y_{5}} \cong$ $\mathcal{O} \oplus \mathcal{O}$ then $d \pi: N_{l / X} \rightarrow N_{\pi(l) / Y_{5}}$ has one-dimensional kernel along $l$. In this case $l$ is contained in the ramification divisor $R$ of $\pi$. The last is possible only for a finite number of $l^{\prime} s$. Therefore, at least for the general tangent line $l \subset S$ to $C$, the line $\pi(l) \subset \pi(S)$ is a $(-1,1)$-line on $Y_{5}$, i.e. $N_{\pi(l) / Y_{5}} \cong \mathcal{O}(-1) \oplus \mathcal{O}(1)$. Therefore $\pi(S) \subset Y_{5}$ coincides with the surface $S_{-1,1} \subset Y_{5}$ swept-out by the $(-1,1)$ lines on $Y_{5}$, which in turn is a tangent scroll to a rational normal sextic (see [FN]). Clearly $\pi: S \rightarrow S_{-1,1}$ is an isomorphism and $S_{-1,1}$ is the tangent scroll to $\pi\left(C_{6}\right)$.

Assume first that $S=R$. Then $S_{-1,1}=\pi(S)=B \cong R$. Therefore $X$ is singular along $C_{6}$ since the branch locus $B=S_{-1,1}$ of $\pi$ is singular along $C_{6}$. In order to prove Lemma (A) for $g=6$ (second kind) it rests to see the general $X_{10}^{\prime} \supset S_{10}$ such that $S \neq R$ is singular. This will imply that any $X_{10}^{\prime} \subset S_{10}$ must be singular-since the property $X_{10}^{\prime} \subset S_{10}$ to have a singularity is a closed condition.

Since $\pi: X_{10}^{\prime} \rightarrow Y_{5}$ is a 2 -sheeted covering with a ramification divisor $R$, then $H^{0}\left(X_{10}^{\prime}, O(1)\right)=\pi^{*} H^{0}\left(Y_{5}, O(1)\right)+C . R$ where $R$ is the hyperplane equation of $R \subset X_{10}^{\prime}$. Therefore, since $S \cong \pi(S)$ and $S \neq R$, then $\pi(S)$ is tangent to the branch locus $B$ of $\pi$ along a (possibly singular) canonical curve $C_{10}^{6}=B \cap H=$ $\pi(S) \cap H$ for some hyperplane $H \subset \boldsymbol{P}^{6}$. Since $\pi(S)=S_{-1,1}$ then the general $X_{10}^{\prime}$ $\supset S=S_{10}, S \neq R$ comes from a branch locus $B$ totally tangent to $S_{-1,1}$ along a general hyperplane section $H \cap S_{-1,1}$. Since $H$ is general then $H$ intersects the rational normal sextic $\pi\left(C_{6}\right)$ at 6 points $p_{1}, \ldots, p_{6}$ such that $p_{i} \neq p_{j}$ for $i \neq j$. By $p_{i} \in B$ and the identification $B=R$, we may cosider $p_{i}$ as points on $R \subset X_{10}^{\prime}$. We shall see that

(*) Lemma. $\quad p_{i} \in \operatorname{Sing} X_{10}^{\prime}, i=1, \ldots, 6$.

Proof of $(*)$. Let $U_{i} \subset Y_{5}$ be a sufficiently small neighborhood of the point $p_{i}$. Since $Y_{5}$ is smooth, we can identify $U_{i}$ with a disk in $C^{3}$, and let $(u, v, w)$ be 
local coordinates in $U_{i}$ s.t. $p_{i}=(0,0,0)$. Let $f(u, v, w)=0$ be the local equation of $S_{10}$ in $U_{i}$. Since $S_{10}$ is the tangent scroll to the smooth rational curve $C_{6}$, and $p_{i} \in C_{6}$, one can choose the coordinates $u, v, w$ such that $f=u^{3}-v^{2}$ (since the scroll $S_{10}$ has a double cusp-singularity along $C_{6}$-see Lemma 2.3). Let $q=0$ and $h=0$ be the local equations of $B$ and $H$ in $U_{i}$. Since $B$ and $S_{10}$ are singular along $C_{10}^{6}=B \cap H=S_{10} \cap H, h^{2}, f$ and $q$ are linearly dependent, i.e. $\alpha h^{2}+\beta f+$ $\gamma q=0$ for some constants $\alpha, \beta, \gamma$. Moreover $\alpha \neq 0$ and $\gamma \neq 0$ since $\pi(S) \cong S$ and $\pi(S) \neq B$, and one may assume that $\beta \neq 0$ (otherwise $B=2 H$ and $X_{10}^{\prime}$ will be singular along the surface $\left.R_{\text {red }} \cong B_{\text {red }}=H\right)$. Since $p_{i} \in H, h(0,0,0)=0$ and $h=$ $a u+b v+c w+o(2)$, where $o(k)$ denotes a sum of terms of degree $\geq k$. Therefore the surface $B$ is singular at $p_{i}$ since $B \cap U_{i}=(q=0)$, and the series expansion

$$
q(u, v, w)=-\beta / \gamma f-\alpha / \gamma h^{2}=\beta / \gamma\left(v^{2}-u^{3}\right)-\alpha / \gamma(a u+b v+c w+o(2))^{2}
$$

has no linear term. Since $B$ is the branch locus of $\pi: X_{10}^{\prime} \rightarrow Y_{5}$, the threefold $X_{10}^{\prime}$ will be also singular at $p_{i} \in R \cong B, i=1, \ldots, 6$. In addition, the $6=12-g\left(X_{10}^{\prime}\right)$ singular points $p_{1}, \ldots, p_{6}$ of $X_{10}^{\prime}$ lie on the rational normal curve $C_{6} \subset X_{10}^{\prime}$.

q.e.d.

\section{Proof of Lemma (A) for $g=8$.}

3.16. The Da Palatini construction (see $[\mathrm{Pu}]$ ). Let $\boldsymbol{P}^{5}=\boldsymbol{P}(V)$ where $V=$ $C^{6}$, and let $\hat{V}=\operatorname{Hom}_{C}(V, C)$ be the dual space of $V$. The points $H \in \wedge^{2} \hat{V}=$ Hom $_{C}\left(\wedge^{2} V, C\right)$ can be regarded as skew-symmetric linear maps $H: V \rightarrow \hat{V}$, and the hyperplanes $(H=0) \subset \boldsymbol{P}^{14}=\boldsymbol{P}\left(\wedge^{2} V\right)$ can be regarded as points of $\hat{\boldsymbol{P}}^{14}=$ $\boldsymbol{P}\left(\wedge^{2} \hat{V}\right)$.

Let $\left.P f=\left\{H \in \wedge^{2} \hat{V}\right): \operatorname{rank}(H) \leq 4\right\} / C^{*}$. Then $P f$ is the Pfaff cubic hypersurface in $\hat{\boldsymbol{P}}^{14}$ defined, in coordinates, by vanishing of the cubic Pfaffian of the skew-symmetric $(6 \times 6)$-matrix $H$. Let $U_{10} \subset \wedge^{2} V$ be a 10-dimensional subspace, and let

$$
\hat{U}_{5}=U_{10}^{\perp}=\left\{H \in \wedge^{2} \hat{V}=\operatorname{Hom}_{C}\left(\wedge^{2} V, C\right):\left.H\right|_{U_{10}}=0\right\} .
$$

Let moreover $U_{10} \subset \wedge^{2} V$ be such that $\operatorname{rank}(H) \geq 4$ for any $H \in U_{10}^{\perp}$, and let $X_{14}=G(2,6) \cap \boldsymbol{P}\left(U_{10}\right)$ and $B_{3}=\operatorname{Pf} \cap \boldsymbol{P}\left(\hat{U}_{5}\right)$.

The construction "Da Palatini" of G. Fano shows that any hyperplane $\boldsymbol{P}^{4} \subset$ $\boldsymbol{P}(V)$ defines a birational isomorphism $\xi: X_{14} \rightarrow B_{3}$ which can be described as follows (see $[\mathrm{Pu}])$ :

Identify the point $b \in B_{3}$ and (the projective equivalence class of) the skewsymmetric $6 \times 6$ matrix corresponding to $b$. Since rank $b=4$ for any $b \in B_{3}$ the projective kernel $n_{b}$ of $b$ will be a line in $\boldsymbol{P}^{5}$. Let $W:=\bigcup_{b \in B_{3}}\left\{n_{b}=\boldsymbol{P}(\operatorname{Ker} b)\right\}$ $\subset \boldsymbol{P}^{5}$.

The fourfold $W$ can be described by an alternative way. Identify the point $l \in G(1: 5)$ and the line $l \subset \boldsymbol{P}^{5}$, and let $W^{\prime}:=\bigcup\left\{l \subset \boldsymbol{P}^{5} \mid l \in X_{14}\right\} \subset \boldsymbol{P}^{5}$. Then (see $[\mathrm{Pu}$, p. 83]): 
(a). for the general $v \in W^{\prime}$ there exists a unique $l \in X_{14}$ such that $v \in l$;

(b). for the general $w \in W$ there exists a unique $b \in B$ such that $w \in n_{b}$;

(c). $W^{\prime}=W$.

Let $H \subset \boldsymbol{P}^{5}$ be a general hyperplane. Then, by (a), (b) and (c), the maps $\phi: X_{14} \rightarrow H \cap V, \phi(l)=H \cap l$ and $\psi: B_{3} \rightarrow H \cap V, \psi(b)=H \cap n_{b}$ are birational isomorphisms. The composition $\xi=\xi_{H}=\psi^{-1} \circ \phi: X_{14} \rightarrow B_{3}$ is a birational isomorphism, depending on the choice of the hyperplane $H \subset \boldsymbol{P}^{5}$ (see [Pu, p. 85]).

3.17. The dual cubic fourfold of $S_{14}$. By Lemma 3.8 (ii) the curve $C_{8}$ is the Plücker image of the tangent scroll to a rational normal quintic $C_{5}:\left(x_{0}: \cdots: x_{5}\right)$ $=\vec{s}=\left(1: s: s^{2}: \cdots: s^{5}\right)$ in $\boldsymbol{P}^{5}(x)$. The points of the curve $C_{8}$ are the Plücker coordinates $x_{i j}(s)$ of the point $\vec{s} \in C_{5}$ :

$$
\left(x_{i j}(s)\right)=\left(\begin{array}{cccccc}
0 & 1 & 2 s & 3 s^{2} & 4 s^{3} & 5 s^{4} \\
\cdots & 0 & s^{2} & 2 s^{3} & 3 s^{4} & 4 s^{5} \\
\cdots & \ldots & 0 & s^{4} & 2 s^{5} & 3 s^{6} \\
\ldots & \ldots & \ldots & 0 & s^{6} & 2 s^{7} \\
\ldots & \ldots & \ldots & \ldots & 0 & s^{8} \\
\ldots & \ldots & \ldots & \ldots & \ldots & 0
\end{array}\right)
$$

Therefore $S_{14}=G(2,6) \cap \boldsymbol{P}^{8}$ where $\boldsymbol{P}^{8}=\left(H_{0}=\cdots=H_{5}=0\right) \subset \boldsymbol{P}^{14}$ and:

$$
\begin{gathered}
H_{0}=x_{03}-3 x_{12}, \quad H_{1}=x_{04}-2 x_{13}, \quad H_{2}=3 x_{05}-5 x_{14}, \\
H_{3}=x_{14}-3 x_{23}, \quad H_{4}=x_{15}-2 x_{24}, \quad H_{5}=x_{25}-3 x_{34} .
\end{gathered}
$$

Let $\Pi^{5}:=\left\langle H_{0}, \ldots, H_{5}\right\rangle \subset \hat{\boldsymbol{P}}^{14}$ be the projective linear span of $\left\{H_{0}, \ldots, H_{5}\right\}$, and let $\boldsymbol{B}_{3}=P f \cap\left\langle H_{0}, \ldots, H_{5}\right\rangle$. Introduce projective coordinates $\left(t_{0}: \cdots: t_{5}\right)$ in $\Pi^{5}$ such that the point $\left(t_{0}, \ldots, t_{5}\right)$ represents the vector $t_{0} H_{0}+\cdots+t_{5} H_{5}$. Then the cubic 4-fold $\boldsymbol{B}_{3}=\operatorname{Pf} \cap \boldsymbol{P}^{5}\left(t_{0}: \cdots: t_{5}\right)$ is defined by $\boldsymbol{B}_{3}: F=32 t_{0} t_{2} t_{5}-t_{0} t_{3} t_{5}-$ $2 t_{1}^{2} t_{5}-2 t_{0} t_{4}^{2}+3 t_{1} t_{3} t_{4}-12 t_{1} t_{2} t_{4}-45 t_{2}^{2} t_{3}-9 t_{2} t_{3}^{2}=0$.

The 6 -vector $(0, \ldots, 0)$ is the only value of $\left(t_{0}, \ldots, t_{5}\right)$ where the 15 Pfaffians $P f_{i j}\left(t_{0}, \ldots, t_{5}\right)$ of the matrix $H\left(t_{0}, \ldots, t_{5}\right)=t_{0} H_{0}+\cdots+t_{5} H_{5}$ vanish. Therefore rank $b=4$ for any $b \in \boldsymbol{B}_{3}$.

The fourfold $\boldsymbol{B}_{3}=(F=0) \subset \Pi^{5}$ is singular, and it is not hard to check

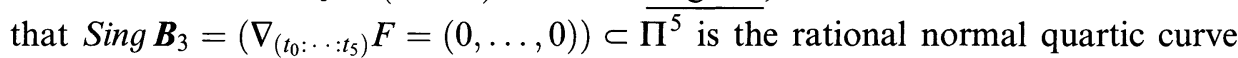
$C_{4}=\left\{[r]=\left(1: 2 r: r^{2} / 3: 8 r^{2} / 3: 2 r^{3}: r^{4}\right) \mid r \in C\right\}$; for simplicity we let $t_{0}=1$.

3.18. Now we are ready to prove Lemma (A) for $g=8$.

Let $X_{14}=G(2,6) \cap \boldsymbol{P}^{9}=\boldsymbol{P}\left(U_{10}\right)$ be such that $X_{14} \supset S_{14}$, and let $B_{3}=$ Pf $\cap \boldsymbol{P}\left(U_{10}^{\perp}\right)$. Then $S_{14} \subset X_{14}=G(2, V) \cap \boldsymbol{P}\left(U_{10}\right) \Leftrightarrow \boldsymbol{P}^{8}=\operatorname{Span} S_{14} \subset \boldsymbol{P}\left(U_{10}\right)=$ Span $X_{14} \Leftrightarrow \boldsymbol{P}\left(U_{10}^{\perp}\right) \subset \Pi^{5} \Leftrightarrow B_{3} \subset \boldsymbol{B}_{3}$. Since $B_{3} \subset \boldsymbol{B}_{3}$ then $\operatorname{rank}(H)=4$ for any $H \in B_{3}$ (see (3.17)), hence the Da Palatini birationalities $\xi: X_{14} \rightarrow B_{3}$ (see (3.16)) are well-defined.

Assume that $X_{14}$ is smooth. Then $B_{3}$ must be smooth (see above or [ $\mathrm{Pu}$, p. 83]). But $B_{3}$ must be singular at any of the intersection points of the hyper- 
plane $\boldsymbol{P}\left(U_{10}^{\perp}\right) \subset \Pi^{5}$ and the rational quartic curve $C_{4}=\operatorname{Sing} \boldsymbol{B}_{3}$-contradiction (see the end of (3.17)). Therefore any $X_{14} \supset S_{14}$ must be singular. q.e.d.

3.19. Remark. If $\boldsymbol{P}\left(U_{10}^{\perp}\right)=\operatorname{Span}\left(C_{4}\right)$ then $B_{3}$ is singular along $C_{4}$, and it can be seen that then $X_{14}$ is singular along $C_{8}$. Let $\boldsymbol{P}\left(U_{10}^{\perp}\right) \neq \operatorname{Span}\left(C_{4}\right)$. Then the hyperplane $\boldsymbol{P}\left(U_{10}^{\perp}\right) \subset \Pi^{5}$ intersects the rational normal quartic $C_{4}=\operatorname{Sing} \boldsymbol{B}_{3}$ in 4 possibly coincident points $b_{1}, b_{2}, b_{3}, b_{4}$. Let, for simplicity $b_{i}$ be different from each other. Then one can show that the general Da Palatini birationality $B_{3} \leftrightarrow X_{14}$ sends $\left\{b_{1}, b_{2}, b_{3}, b_{4}\right\}$ to $4=12-g\left(X_{14}\right)$ singular points of $X_{14}$ which lie on $C_{8}$. Let $H \subset \boldsymbol{P}^{5}$ be a hyperplane, and let $\xi_{H}^{-1}: B_{3} \rightarrow X_{14}$ be the Da Palatini birationality defined by $H$. We shall see that for the general $H$ the rational map $\xi_{H}^{-1}=\psi_{H}$ is regular at a neighborhood of any $b_{i}$, and $\xi_{H}^{-1}\left(b_{i}\right) \in C_{8}$. For this, by the definition of the maps $\phi$ and $\psi$, it is necessary to see that the kernel-map ker : $B_{3} \rightarrow G(1: 5), b \mapsto n_{b}$ sends the quartic $C_{4}$ isomorphically to $C_{8}$.

Let $b=\left(b_{i j}\right) \in B_{3}$. Then the Plücker coordinates of the line $n_{b}=\operatorname{ker}(b)$ are $(-1)^{l+J} P f_{i j}(b)$, where $P f_{i j}(b)$ are the 15 quadratic Pfaffians of the skew-symmetric matrix $\hat{b}$; note that $\operatorname{rank}(b)=4$ for any $b \in B_{3} \subset \boldsymbol{B}_{3}$. Now, it rests only to replace $b$ by the general point $b(t)=H_{0}+t H_{1}+t^{2} / 12 H_{2}+2 t^{2} / 3 H_{3}+t^{3} / 4 H_{4}+$ $t^{4} / 16 H_{5} \in C_{4}$, and to see that the Plücker coordinates of $b(t)$ parameterize the general point $x_{i j}(\vec{s})$ of $C_{8}$ (where $s=2 / t$ ) - see (3.17).

\section{Proof of Lemma (A) for $g=7$}

3.20. In the proof of Lemma (A) for $g=7$ we shall need the known by [I2] description of the projection from a line $l$ on a smooth prime Fano threefold $X_{2 g-2}$ such that $N_{l / X}=\mathcal{O}(1) \oplus \mathcal{O}(-2)$.

\subsection{1.}

LEMMA (see $\S 1$ Proposition 3 in [I2]). Let $l$ be a line on the smooth prime Fano threefold $X=X_{2 g-2} \subset \boldsymbol{P}^{g+1}$ such that $N_{l / X}=\mathcal{O}_{l}(-2) \oplus \mathcal{O}_{l}(1)$, and let $\sigma: X^{\prime} \rightarrow X$ be the blow up of $l$. Let $Z^{\prime}=\sigma^{-1}(l)$, let $H^{\prime} \sim \sigma^{*} H-Z^{\prime}$ be the proper preimage of the hyperplane section $H$ of $X$, and let $\pi: X \rightarrow X^{\prime \prime} \subset \boldsymbol{P}^{g-1}$ be the projection from $l$. Then:

(i). If $g \geq 5$ then the composition $\phi=\pi \circ \sigma: X^{\prime} \rightarrow \boldsymbol{P}^{g-1}$ is a birational morphism (given by the linear system $\left|H^{\prime}\right|$ ), to a threefold $X^{\prime \prime} \subset \boldsymbol{P}^{g-1}$.

(ii). The restriction to $Z^{\prime}=\boldsymbol{P}\left(N_{l / V}\right)=\boldsymbol{F}_{3}$ of the linear system $\left|H^{\prime}\right|$ is the complete linear system $\left|s^{\prime}+3 f^{\prime}\right|$, where $s^{\prime}$ and $f^{\prime}$ are the classes of the exceptional section and the fiber of the rational ruled surface $Z^{\prime}$.

(iii). The restriction $\left.\phi\right|_{Z^{\prime}}$ of $\phi$ to $Z^{\prime}$ maps $Z^{\prime}$ to a cone $Z^{\prime \prime}$ over a twisted cubic curve, contracting the exceptional section $s^{\prime}$ of $Z^{\prime}$ to the vertex of $Z^{\prime \prime}$.

(iv). If $g \geq 7$ then there are only a finite number of lines $l_{i} \subset X(i=1, \ldots, N)$ which intersect l. Let $l_{1}^{\prime} \subset X^{\prime}$ of $l_{i}(i=1, \ldots, N)$ be the proper preimages $l_{i}^{\prime} \subset X^{\prime}$ of $l_{i}(i=1, \ldots, N)$. Then the morphism $\phi: X^{\prime} \rightarrow X^{\prime \prime}$ is an isomorphism outside $l_{1}^{\prime} \cup \cdots \cup l_{N}^{\prime} \cup s^{\prime}$, and $\phi$ contracts $s^{\prime}$ and any of $l_{l}^{\prime}$ to isolated double points of $X^{\prime \prime}$.

(v). Let $H^{\prime \prime}=\phi\left(H^{\prime}\right)$ be the hyperplane section of $X^{\prime \prime}$. Then if $g \geq 7$ then $-K_{X^{\prime \prime}} \sim H^{\prime \prime}$, i.e. the variety $X^{\prime \prime}=X_{2(g-2)-2}^{\prime \prime} \subset \boldsymbol{P}^{g-1}$ is an anticanonically embedded Fano threefold with isolated singularties as in (iv). 
3.22. Suppose that there exists a smooth prime $X=X_{12} \subset \boldsymbol{P}^{8}$ which contains the tangent scroll $S_{12}$ to the rational normal curve $C_{7}$. Therefore the general such $X$ is smooth, and we may suppose that $X \supset S_{12}$ is general.

Let $l \subset S_{12}$ be any of the tangent lines to $C_{7}$ and let $\pi: X \rightarrow X^{\prime \prime}$ be the projection from $l$. By [I2, §1] (see also (1.2)) $N_{l / X}=\mathcal{O}(1) \oplus \mathcal{O}(-2)$, therefore Lemma 3.21 (i) $-(\mathrm{v})$ take place.

By Lemma $3.21(\mathrm{v})$ the threefold $X^{\prime \prime}=X_{8}^{\prime \prime} \subset \boldsymbol{P}^{6}$ is an anticanonically embedded Fano threefold of genus 5. Let $S^{\prime \prime} \subset X^{\prime \prime}$ be the proper image of $S_{12}$, and let $C^{\prime \prime} \subset S^{\prime \prime} \subset X^{\prime \prime}$ be the proper image of $C_{7}$. It is easy to see that $C^{\prime \prime}=C_{5}^{\prime \prime}$ is a rational normal quintic, and $S^{\prime \prime}=S_{8}^{\prime \prime} \subset X^{\prime \prime}$ is the tangent scroll to $C^{\prime \prime}$.

In order to use the proof of Lemma (A) for $g=5$ we have to see whether $X_{8}^{\prime \prime} \subset \boldsymbol{P}^{6}$ is, in fact, a complete intersection of three quadrics. If $X^{\prime \prime}$ were nonsingular then the classification of the smooth Fano threefolds will imply that $X^{\prime \prime}$ will be a complete intersection of three quadrics. But $X^{\prime \prime}$ is singularsee Lemma 3.21 (iv),(v).

However, especially in this case, $X^{\prime \prime}=X_{8}^{\prime \prime} \subset \boldsymbol{P}^{6}$ is still a complete intersection of three quadrics (see Theorem 6.1 (vii) in [I1]).

Denote by $\operatorname{Sing}(X) \cap C$ the set of singular points of $X=X_{12}$ on $C$, and let $\operatorname{Sing}\left(X^{\prime \prime}\right) \cap C^{\prime \prime}$ be the set of singular points of $X^{\prime \prime}$ on $C^{\prime \prime}$.

3.23. By the proof of Lemma (A) for $g=5$, the elements of $\operatorname{Sing}\left(X^{\prime \prime}\right) \cap C$ are in a $(1: 1)$ correspondence with the different zeros of a homogeneous form $F_{7}\left(s_{o}: s_{1}\right)$ of degree 7. (see (3.6)). Clearly, the vertex $o$ of $Z^{\prime \prime}$ lies on $C^{\prime \prime}$. Moreover, by (3.21)(iv), $o$ is a double singularity of $X^{\prime \prime}$. Since $l \cap \operatorname{Sing} X=\emptyset$, and since the tangent lines $l^{\prime} \neq l$ to $C=C_{7}$ do not intersect $l,(3.21)$ (iv) yield that, set-theoretically: $\operatorname{Sing}(X) \cap C \cong \operatorname{Sing}\left(X^{\prime \prime}\right) \cap C^{\prime \prime}-\{o\}$.

Let $F_{7}\left(s_{0}: s_{1}\right)=0$ be as above. Since $\operatorname{dim} \operatorname{Sing} X^{\prime \prime}=0$, the form $F_{7}$ does not vanish on $C_{7}$; and we can assume that $o=(1: 0)$ and $F_{7}(0: 1) \neq 0$. Therefore if $s=s_{1} / s_{0}$ and $f_{7}(s)=F(1: s)$ then $\operatorname{deg} f_{7}(s)=7$. By the previous the elements of $\operatorname{Sing}(X) \cap C$ correspond to the different zeros of the polynomial $s^{-m} \cdot f_{7}(s)=0$, where $m=$ mult $_{o} f_{7}(s)$.

By the local definition of $m$, the integer $m=m(o)=$ mult $_{o} f_{7}(s)$ does not depend on the genus $g \geq 7$ of $X_{2 g-2}$ as well on the choice of the general tangent line $l$ to $C_{g}$. It can be seen that $m=2$, but for the proof it is enough to know that $m \leq 2$.

\section{(*) Lemma. $m \leq 2$.}

Proof of $(*)$. By construction $X^{\prime \prime} \supset S^{\prime \prime} \cup Z^{\prime \prime}$ where $S^{\prime \prime}=S_{8}^{\prime \prime}$ is the tangent scroll to the rational normal quintic $C^{\prime \prime}=C_{5}^{\prime}$ such that $o \in C^{\prime \prime}, Z^{\prime \prime}$ is a cone over a twisted cubic, and $o$ is the vertex of $Z$. Moreover $Z^{\prime \prime}$ is triple tangent to $S^{\prime \prime}$ at the tangent line $F$ to $C^{\prime \prime}$ at $o$. Indeed $S^{\prime \prime}$ is a hyperplane section of $Z^{\prime \prime}$ which passes through the vertex $o$ of $Z$. Therefore $S^{\prime \prime} . Z=f_{1}+f_{2}+f_{3}$ is a sum of 3 rulings of $Z^{\prime \prime}$. Since $f_{i}$ are rulings of $Z, o \in f_{i}$ for $i=1,2,3$. Therefore any $f_{i}$ is a line on the tangent scroll $S^{\prime \prime}$ to $C^{\prime \prime}$ which passes through $o \in C^{\prime \prime}$. Therefore $f_{i}=F$ must be a tangent line to $C^{\prime \prime}$ at $o$, i.e. $S^{\prime \prime} . Z^{\prime \prime}=3 F$. 
By Theorem 9.9 in [I3], the general complete intersection $X_{8} \subset \boldsymbol{P}^{6}$ of three quadrics, containing a cone $Z_{3}$ over a twisted cubic, is a projection of $X_{12}$ from a line $l$ such that $N_{l / X_{12}}=\mathcal{O}(1) \oplus \mathcal{O}(-2)$. The inverse of the projection $\pi_{l}$ is defined by the linear system $\left|H+Z_{3}\right|$, where $H$ is the hyperplane section of $X_{8}$.

Let $X_{8} \supset Z_{3} \cup S_{8}$ be as above. Then $X_{12}$ will contain a tangent scroll $S_{12}$ to a rational normal curve $C_{7}$, and $l$ will be a tangent line to $C_{7}$. Therefore any $X_{8} \supset Z_{3} \cup S_{8}$ will be a deformation of a projection of $X_{12} \subset S_{12}$ from a tangent line to $C_{7}$.

It rests to see that $m\left(X_{8}\right)=$ mult $_{o} f_{7} \leq 2$ for $f_{7}$ corresponding, as above, to some particular such $X_{8}$.

Example. Let $\boldsymbol{P}^{5}(x)=\boldsymbol{P}^{5}\left(x_{0}: \cdots: x_{5}\right)$, and let $q_{0}=-x_{0} x_{4}+4 x_{1} x_{3}-3 x_{2}^{2}$, $q_{1}=-x_{0} x_{5}+3 x_{1} x_{4}-2 x_{2} x_{3}, q_{2}=-x_{1} x_{5}+4 x_{2} x_{4}-3 x_{3}^{2}$. Then $S_{8}=\left(q_{0}=q_{1}=\right.$ $\left.q_{2}=0\right) \subset \boldsymbol{P}^{5}(x)$ will be the tangent scroll to the rational normal quintic $C_{5}$ : $x_{l}=s_{0}^{5-\imath} s_{1}^{l}(0 \leq i \leq 5)$.

Let $X_{8}=\left(Q_{0}=Q_{1}=Q_{2}=0\right) \subset \boldsymbol{P}^{6}(x: u)=\boldsymbol{P}^{6}\left(x_{0}: x_{1}: x_{2}: x_{3}: x_{4}: x_{5}: u\right)$, where

$$
\begin{aligned}
& Q_{0}=q_{0}+L_{o}\left(x_{4}: x_{5}\right) u \\
& Q_{1}=q_{1}+\left(12 x_{1}+L_{1}\left(x_{4}: x+5\right)\right) u \\
& Q_{2}=q_{2}+\left(27 / 2 x_{2}+L_{2}\left(x_{4}: x_{5}\right)\right) u
\end{aligned}
$$

$L_{0}, L_{1}$ and $L_{2}$ being linear forms of $\left(x_{4}: x_{5}\right)$. Evidently $X_{8} \cap(u=0)=S_{8}$.

Let $\boldsymbol{P}^{4}=\boldsymbol{P}^{4}\left(x_{0}: x_{1}: x_{2}: x_{3}: u\right) \subset \boldsymbol{P}^{6}$, and let $Z_{3}=X_{8} \cap \boldsymbol{P}^{4}$. Then $Z_{3}=$ $\left(P_{0}=P_{1}=P_{2}=0\right) \subset P^{4}$, where $P_{0}=x_{1} x_{3} / 3-x_{2}^{2} / 4, P_{1}=x_{1} u-x_{2} x_{3} / 6, P_{2}=$ $x_{2} u-x_{3}^{2} / 9$.

Therefore $Z_{3}$ is a cone with center $o=(1: 0: \cdots: 0) \in C_{5}$ over the twisted cubic curve $C_{3}=Z_{3} \cap\left(x_{0}=0\right), C_{3}:\left(x_{1}: x_{2}: x_{3}: u\right)=\left(t_{0}^{3}: 2 t_{0}^{2} t_{1}: 3 t_{0} t_{1}^{2}: t_{1}^{3}\right)$. Let $s=s_{1} / s_{0}$, and we may suppose that the point $(0: \cdots: 0: 1) \in C_{5}$ is not a singular point of $X_{8}$. Then, by (3.6), the equation of $\left.\left(\operatorname{Sing} X_{8}\right)\right|_{C_{5}}$ is

$$
\begin{aligned}
f_{7}(s) & =s^{2} \partial Q_{0} / \partial u\left(1: s: \cdots: s^{5}\right)-s \partial Q_{1} / \partial u\left(1: s: \cdots: s^{5}\right)+\partial Q_{2} / \partial u\left(1: s: \cdots: s^{5}\right) \\
& =s^{2} L_{0}\left(s^{4}, s^{5}\right)-s\left(12 s+L_{1}\left(s^{4}, s^{5}\right)\right)+\left(27 / 2 s^{2}+L_{2}\left(s^{4}, s^{4}\right)\right)=3 / 2 s^{2}+o\left(s^{3}\right),
\end{aligned}
$$

where $o\left(s^{3}\right)$ is a sum of terms of degree $\geq 3$. Therefore $m\left(X_{8}\right)=$ mult $_{o} f_{7}(s)=2$. q.e.d.

3.24. Let $X=X_{12} \supset S_{12}$ be general. Since $m \leq 2$ then $\operatorname{deg} s^{-m} f_{7}(s) \geq 7-$ $2=5=12-g\left(X_{12}\right)>0$. In particular $g(s):=s^{-m} f_{7}(s)$ is not a constant. Since $g(0) \neq 0$, and since the elements of $\operatorname{Sing}(X) \cap C$ are in a $(1: 1)$ correspondence with the different zeros of $g(s)=s^{-m} f_{7}(s)$ (see above), then $X_{12}$ must be singular, which contradicts the initial assumption. This proves Lemma (A) for $g=7$. 


\section{Proof of Lemma (A) for $g=9$.}

3.25. Let $X_{16} \subset \boldsymbol{P}^{10}$ contains the tangent scroll $S=S_{16}$ to the rational normal curve $C=C_{9}$, and suppose that nevertheless $X_{16}$ is smooth.

Let $L \subset X_{16}$ be a tangent line to $C$, and consider the double projection $\pi=$ $\pi_{2 L}$ of $X$ from the line $L$, i.e. $\pi$ is the rational map on $X$ defined by the noncomplete linear system $\left|\mathcal{O}_{X}(1-2 L)\right|$. Since $X=X_{16}$ is assumed to be smooth then, by $\S 2$ in [I2]:

(*). $\pi=\pi_{2 L}$ sends $X$ birationally to $\boldsymbol{P}^{3}$. Moreover, on $\boldsymbol{P}^{3}$ there exists a smooth irreducible curve $C=C_{7}^{3}$ of genus 3 and degree 7 , which lies on a unique cubic surface $S_{3} \subset \boldsymbol{P}^{3}$, and such that the inverse to $\pi$ birational map $\phi: \boldsymbol{P}^{3} \rightarrow X$ is given by the non-complete linear system $\left|\mathcal{O}_{p^{3}}(7-2 C)\right|$.

By $(*)$, the proper image $\pi(H)$ of any hyperplane section $H \subset X$ is an irreducible component of an effective divisor $S_{7} \in\left|\mathcal{O}_{P^{3}}(7-2 C)\right|$. If moreover $H$ contains the line $L$ but $H \notin\left|\mathcal{O}_{X}(1-2 L)\right|$ (for example if $\left.H=S_{16}\right)$ then $\pi(H) \subset$ $\boldsymbol{P}^{3}$ will be a quartic surface containing the curve $C=C_{7}^{3}$ (see the proof of the Main Theorem in $\S 2$ of [I2]), and in this case $S_{7}=\pi(H)+S_{3} \in\left|\mathcal{O}_{P^{3}}(7-2 C)\right|$.

Therefore $S_{4}:=\pi\left(S_{16}\right)$ is a quartic surface in $\boldsymbol{P}^{3}$ containing the curve $C=$ $C_{7}^{3}$. Moreover, the double projection $\pi$ sends the general tangent line $L^{\prime}$ to $C_{9}$ to a tangent line $\pi\left(L^{\prime}\right)$ to the proper image $\pi\left(C_{9}\right)$; and since $C_{9} \cong \boldsymbol{P}^{1}$ then $\pi\left(C_{9}\right)$ is rational. Therefore the quartic surface $S_{4}=\pi\left(S_{16}\right)$ is the tangent scroll to the rational curve $\pi\left(C_{9}\right) \subset \boldsymbol{P}^{3}$. The last is only possible if $\pi\left(C_{9}\right)=C_{3}$ is a twisted cubic and $S_{4}$ is the tangent scroll to $C_{3}$, and we shall see that this is impossible.

The surface $S_{4} \subset \boldsymbol{P}^{3}$ is the tangent scroll to the twisted cubic $C_{3}$. Then, by Lemma 1.6 and p. 498 in [MU], the normalization of $S_{4}$ is the quadric $\boldsymbol{P}^{1} \times \boldsymbol{P}^{1}$, and the map $v: \boldsymbol{P}^{1} \times \boldsymbol{P}^{1} \rightarrow S_{4}$ is given by a linear system of bidegree $(1,2)$.

Let $\Gamma \subset \boldsymbol{P}^{1} \times \boldsymbol{P}^{1}$ be the proper transform of $C_{7}^{3}$, and let $(a, b)$ be the bidegree of $\Gamma$. Therefore $7=\operatorname{deg}\left(C_{7}^{3}\right)=2 a+b$, and $3=g\left(C_{7}^{3}\right)=g(\Gamma)=(a-1)(b-1)$. Obviously, these two equations for the integers $a$ and $b$ have no integral solutions-contradiction. $g=9$.

Therefore $X_{16} \supset S_{16}$ can't be smooth, which proves Lemma (A) in case This completes the proof of Lemma (A).

\section{REFERENCES}

[AS] E. Arrondo and I. Sols, On congruences of lines in the projective space, Mémoire no. 50, Supplément au Bulletın de la SMF, Tome 120, fasc. 3 (1992).

[FN] M. Furushima AND N. Nakayama, The family of lines on the Fano threefold $V_{5}$, Nagoya Math. J., 116 (1989), 111-122.

[FT] M. Furushima AND M. TADA, Non-normal del Pezzo surfaces and Fano threefolds of the first kınd, J. Reine Angew. Math., 429 (1992), 183-190.

[GH] P A. Griffiths and J. Harris, Principles of Algebraic Geometry, Willey, 1978. 
[Gu] N. P GuShel, Fano varieties of genus 6, Math. USSR-Izv., 21 (1983), 445-459.

[H] R. HARTSHORNe, Algebralc Geometry, Sprınger-Verlag, 1977

[I1] V Iskovskikh, Fano threefolds II, Math. USSR-Izv., 12 (1978), 469-506.

[I2] V Isкovsкiкh, Double projection from a line on Fano threefolds of the first kınd, Math. USSR-Sb., 66 (1990), 265-284.

[13] V IskovskikH, Lectures on Three-Dimensional Algebraic Varieties. Fano varieties, Moscow Univ. Publ., 1988, (in Russian).

[IP] V Iskovskikh and Yu. Prokhorov, Algebraic Geometry V· Fano Varıeties, SpringerVerlag, 1999.

[L] R. LAZARSFeld, Some applications of the theory of positive vector bundles, Lect. Notes in Math. 1092, Springer-Verlag, 1984, 29-61.

[M1] S. MukaI, Curves, $K 3$ surfaces and Fano 3-folds of Genus $\leq 10$, Algebra1c Geometry and Commutatıve Algebra Volume I (H. Hijikata et al eds.), Kinokunıya, 1987, 357-377

[M2] S. MukaI, Fano 3-folds, London Math. Soc. Lect. Note Ser. 179, Cambridge, 1992, 255263.

[MU] S. Mukai and H. Umemura, Minimal rational threefolds, Lect. Notes in Math. 1016, Springer-Verlag, 1983, 490-518.

[P1] R. PIENE, Some formulas for a surface in $\boldsymbol{P}^{3}$, Lect. Notes in Math. 687, Springer-Verlag, 1978, 196-235.

[P2] R. PiEnE, Cuspidal projections of space curves, Math. Ann., 256 (1981), 95-119.

[Pe] T. Peternell, Manifolds of semi-positive curvature, Lect. Notes in Math. 1646, SpringerVerlag, 1996, 98-142.

[Pr] Yu. Prokhorov, Exotic Fano varieties, Moscow Univ. Math. Bull., 45 (1990), $36-38$.

[PS] K. ParanjaPe AND V SRInivas, Self maps of homogeneous spaces, Invent. Math., 98 (1989), 425-444.

[Pu] P J. Puts, On some Fano threefolds that are sections of Grassmannians, Proc. Konınk. Nederl. Acad. Wetensch., 85 (1982), 77-90.

[RV] R. REMmERT, A. VAN DE VEN, Zweı Sätze über die komplex-projectıve Ebene, Nieuw Archief voor de Wiskunde (3) VIII (1960), 147-157

[Sch] C. Schummann, Morphisms between Fano threefolds, J. Algebr. Geom., 8 (1999), 221-244.

[Sh] I. R. Shafarevich, Basic Algebraic Geometry, Springer-Verlag, 1977

INSTITUTE OF MATHEMATICS,

Bulgarian ACADEMy OF SCIENCES

ACAD. G. Bonchev Str. 8, 1113 Sofia,

BULGARIA

University of Leiden, P.O. Box 9512

2300 RA LEIDEN,

THE NETHERLANDS 